How good is quantized model predictive control with horizon one?

by Claus Müller, Daniel E. Quevedo, \& Graham C. Goodwin

Copyright (C) 2011 IEEE.

This is an author-prepared version of the article, reprinted from IEEE Transactions on Automatic Control, Vol. 56, Issue 11, p. 2623-2638.

http://dx.doi.org/10.1109/TAC.2011.2122610

This material is posted here with permission of the IEEE. Such permission of the IEEE does not in any way imply IEEE endorsement of any of University of Newcastle's products or services. Internal or personal use of this material is permitted. However, permission to reprint/republish this material for advertising or promotional purposes or for creating new collective works for resale or redistribution must be obtained from the IEEE by writing to pubs-permissions@ieee.org. By choosing to view this document, you agree to all provisions of the copyright laws protecting it. 


\title{
How Good is Quantized Model Predictive Control with Horizon One?
}

\author{
Claus Müller, Daniel E. Quevedo, Member, IEEE, Graham C. Goodwin, Fellow, IEEE
}

\begin{abstract}
Model Predictive Control is increasingly being used in areas where decision variables are constrained to finite or countably infinite sets. Well known fields include Power Electronics, Signal Processing, and Telecommunications. Typically, the applications utilize high speed sampling and, thus, there is an incentive to reduce computational burden. One way of achieving this is to use small optimization horizons. This raises the question as to the optimality and performance of control laws with short horizons. In this paper, we give necessary and sufficient conditions for horizon one quantized model predictive control to be equivalent to the use of larger horizons. We also explore situations where horizon one is near optimal.
\end{abstract}

\section{INTRODUCTION}

Model Predictive Control (MPC) is having a major impact on advanced control applications [1]-[5]. Initially, the focus was mainly on process control problems having relatively slow sampling rates. However, there has also been a recent trend towards high speed applications, including the design of switching laws for power electronics and drives [6]-[8], subband coding in filter banks [9], and equalization of digital communication channels [10].

A special feature of these applications is that the input signal is constrained to take only a finite or countably infinite number of values; e.g, $\pm 1 \mathrm{~dB}$ in the case of inner loop power control of cellular communication systems [11], or finite switching states in power electronics. Unfortunately, the associated optimization problems are inherently non-convex and the computational burden grows, in general, exponentially with the MPC optimization horizon. Thus, there is a strong incentive to use small horizons. For example, in the area of Power Electronics and Drives, almost all applications use a horizon of length one, see [6].

This raises an important question: Can horizon one quantized MPC ever be equivalent to larger horizon quantized MPC? This would clearly be an important observation, if it were true. A second question is that of quantifying the degree of sub-optimality, in case horizon one quantized MPC is not optimal. By way of background to the above question, we note that necessary and sufficient conditions are known such that horizon one MPC is equivalent to larger horizon optimizations in cases when inputs are constrained to belong to a convex set [12]. The situation when the inputs are constrained to belong to finite or countably infinite sets is much more difficult.

In this paper we give necessary and sufficient conditions for horizon one MPC to be equivalent to horizon $N>1$

The authors are with the School of Electrical Engineering \& Computer Science, The University of Newcastle, Callaghan, NSW 2308, Australia; Emails: claus.mueller@newcastle.edu.au, dquevedo@ieee.org, graham.goodwin@newcastle.edu.au
MPC when the input is constrained to a countably infinite set. Preliminary results of this type have been established by the present authors in [13], [14]. The present paper goes beyond [13], [14] by developing both necessary, and necessary and sufficient conditions for optimality in the case of plants of arbitrary order. The key novelty of the present work lies in that we adopt a geometric analysis framework of the lattice vector quantizers underlying the optimal solutions. We also explore cases where horizon one quantized MPC gives nearly the same performance as larger horizon optimizations. The results are important in a range of applications since they give reassurance to the use of horizon one MPC.

The remainder of this work is organized as follows: In Section II, we give some background on MPC with quantized inputs and formulate the question of optimality to be studied in precise terms. Optimality is then treated for general situations in Section III. Section IV then analyzes more specific cases. Section V examines robustness of the results to small changes in the system parameters. Examples are included in Section VI. Section VII draws conclusions.

Notation: The superscript ${ }^{T}$ refers to transpose. The set of integers is denoted via $\mathbb{Z}$ and we define $\mathbb{N}^{+}:=\{1,2, \ldots\}$ and $\mathbb{N}_{0}:=\{0\} \cup \mathbb{N}^{+}$. The symbol $\lfloor\cdot\rfloor$ stands for the Gaussbracket; that is, $\lfloor r\rfloor$ is the largest integer less or equal than $r$. $E_{n}$ denotes the $n \times n$ identity matrix. The symbol $e_{k, N}$ denotes the $k$-th unit vector in $\mathbb{R}^{N}$; for example, $e_{1, N}^{T}=(1,0, \ldots, 0)$. The symbol $|\cdot|$ stands for the Euclidean norm; $\|\cdot\|$ denotes any matrix norm compatible with the Euclidean norm. In particular, $\|\cdot\|_{2}$ refers to the matrix norm induced by the Euclidean norm. The linear hull is denoted by Span. We also recall that the linear hull of the empty set $\emptyset$ satisfies $\operatorname{Span} \emptyset=\{0\}$. For any set $X, \partial X$ denotes the boundary of $X$, i.e, the closure without the interior: $\partial X=\bar{X} \backslash X^{o}$. The superscript ${ }^{\perp}$ denotes the orthogonal complement. Throughout this work, we write 'iff' instead of 'if and only if'.

\section{MPC WITH QUANTIZED InPUTS}

We will focus on systems described in discrete time via

$$
x(\ell+1)=A x(\ell)+B u(\ell), \quad \ell \in \mathbb{N}_{0},
$$

where $A \in \mathbb{R}^{n \times n}$ and $B \in \mathbb{R}^{n}$ are both nonzero, and $x(\ell) \in$ $\mathbb{R}^{n}$ denotes the system state at time instant $\ell$. A key feature of the problem of interest in this paper is that the plant input in (1) is quantized to a countably infinite set. More precisely, it is required to satisfy:

$$
\begin{gathered}
u(\ell) \in \mathbb{U}, \quad \forall \ell \in \mathbb{N}_{0}, \\
\mathbb{U}=\delta \mathbb{Z}=\{0, \pm \delta, \pm 2 \delta, \ldots\},
\end{gathered}
$$

for a given stepsize $\delta>0$. 


\section{A. Receding Horizon Optimization}

When evaluating the input for the system (1) via MPC with quantized inputs (as presented in [15], [16]), at every time instant $\ell \in \mathbb{N}_{0}$, and given the current plant state, say $x(\ell)=$ $x_{0}$, the following quadratic cost function is minimized:

$$
V_{N}\left(x_{0}, \vec{u}^{\prime}\right)=\left(x_{N}^{\prime}\right)^{T} P\left(x_{N}^{\prime}\right)+\sum_{j=0}^{N-1}\left(x_{j}^{\prime}\right)^{T} Q\left(x_{j}^{\prime}\right)+R\left(u_{j}^{\prime}\right)^{2} .
$$

In the above cost function,

$$
\vec{u}^{\prime}:=\left(u_{0}^{\prime}, \ldots, u_{N-1}^{\prime}\right)^{T}
$$

contains the decision variables, $N \geq 1$ is the prediction horizon, $R \in[0,+\infty)$, whereas $P, Q \in \mathbb{R}^{n \times n}$ are positive definite matrices. The cost in (4) examines predictions of the future plant state trajectory. These depend upon (1) and $\vec{u}^{\prime}$, and are calculated via the prediction model:

$$
x_{j+1}^{\prime}=A x_{j}^{\prime}+B u_{j}^{\prime}, \quad 0 \leq j<N,
$$

where $x_{0}^{\prime}=x_{0}$ is the initial state. In accordance with (2), the control sequence $\vec{u}$ is only allowed to take values in the quantized set

$$
\mathbb{U}^{N}:=\mathbb{U} \times \mathbb{U} \times \cdots \times \mathbb{U} .
$$

Whilst the set of optimal control sequences, say

$$
\vec{u}=\arg \min _{\vec{u}^{\prime} \in \mathbb{U}^{N}} V_{N}\left(x_{0}, \vec{u}^{\prime}\right),
$$

contains control inputs which are designed for time instants $\{\ell, \ell+1, \ldots, \ell+N-1\}$, following the receding horizon optimization principle, see, e.g., [17], only the first element of $\vec{u}$ is implemented, i.e., we have:

$$
u(\ell) \in e_{1, N}^{T} \vec{u} .
$$

At the next sampling instant, namely $\ell+1$, the state $x(\ell+1)$ is used to carry out another optimization, with initial state $x_{0}=x(\ell+1)$. This yields $u(\ell+1)$. The above procedure is repeated on-line and ad infinitum.

\section{B. Closed Form Solution}

In the sequel, we will utilize a known expression for the optimizer $\vec{u}$, which was presented originally in [15]. To state this result, we introduce the matrices

$$
\begin{gathered}
\Phi_{N}=\left(\begin{array}{ccccc}
B & 0 & \ldots & \ldots & 0 \\
A B & B & 0 & \ldots & 0 \\
\vdots & \vdots & \vdots & \vdots & \vdots \\
A^{N-1} B & \ldots & \ldots & A B & B
\end{array}\right) \in \mathbb{R}^{N n \times N}, \\
\widehat{Q}_{1}=P, \widehat{Q}_{N}=\left(\begin{array}{cccc}
Q & 0 & \ldots & 0 \\
\vdots & \ddots & \ddots & \vdots \\
0 & \ldots & Q & 0 \\
0 & \ldots & 0 & P
\end{array}\right) \in \mathbb{R}^{N n \times N n}, \\
\widehat{R}_{N}=\left(\begin{array}{cccc}
R & 0 & \ldots & 0 \\
0 & R & \ldots & 0 \\
\vdots & \ddots & \ddots & \vdots \\
0 & \ldots & 0 & R
\end{array}\right) \in \mathbb{R}^{N \times N}, \quad \Lambda_{N}=\left(\begin{array}{c}
A \\
A^{2} \\
\vdots \\
A^{N}
\end{array}\right)
\end{gathered}
$$

and also define the positive definite $(N \times N)$-matrix $H_{N}$ and the $(N \times n)$-matrix $F_{N}$ via:

$$
\begin{aligned}
H_{N} & :=\Phi_{N}^{T} \widehat{Q}_{N} \Phi_{N}+\widehat{R}_{N}, \\
F_{N} & :=\Phi_{N}^{T} \widehat{Q}_{N} \Lambda_{N} .
\end{aligned}
$$

Finally, let $\mathcal{P}\left(\mathbb{R}^{N}\right)$ be the power set of $\mathbb{R}^{N}$ and define the multi-valued projection:

$$
q_{\widetilde{\mathbb{U}}^{N}}: \mathbb{R}^{N} \rightarrow \mathcal{P}\left(\mathbb{R}^{N}\right)
$$

by $x \in q_{\widetilde{\mathbb{U}}^{N}}(y)$, iff $x \in \widetilde{\mathbb{U}}^{N}:=H_{N}^{1 / 2} \mathbb{U}^{N}$ and $|x-y| \leq|z-y|$ for all $z \in \widetilde{\mathbb{U}}^{N}$. Note that $q_{\widetilde{\mathbb{U}}^{N}}(\cdot)$ amounts to a (multi-valued) lattice vector quantizer; see, e.g., [18].

Theorem 1 in [15] states that the optimizer (7) is provided by the multi-valued mapping:

$$
\vec{u}=H_{N}^{-1 / 2} q_{\widetilde{\mathbb{U}}^{N}}\left(-H_{N}^{-1 / 2} F_{N} x_{0}\right)
$$

and hence the quantized MPC law, see (8), can be expressed as the static non-linear mapping

$$
\begin{gathered}
u(\ell) \in \kappa_{N}(x(\ell)), \\
\kappa_{N}(x):=e_{1, N}^{T} H_{N}^{-1 / 2} q_{\widetilde{\mathbb{U}}^{N}}\left(-H_{N}^{-1 / 2} F_{N} x\right) .
\end{gathered}
$$

Choosing larger horizons $N$ in the cost function will, in general, give better closed loop performance than choosing short horizons; see, e.g., [9], [10], [19]. Unfortunately, obtaining the optimizer(s) in (11) requires that one solve a combinatorial optimization whose search space is countably infinite [18] and which impedes the use of large horizons in practical applications. The purpose of the current paper is to obtain conditions under which the horizon one version of the control law (13) is equivalent, or nearly equivalent, to the horizon $N$ case, where $N>1$. Our analysis will make use of geometric properties of the partition induced by the projection defined in (10).

\section{The Horizon One Law}

As an alternative to calculating the optimizing sequences $\vec{u}$ via (11), in the present work we will study the horizon one version. The horizon one controller is obtained by setting $N=1$ in (13) and, therefore, has the special form:

$$
\kappa_{1}(x)=q_{\mathbb{U}}\left(-B^{T} P A x / \gamma\right), \quad \gamma:=B^{T} P B+R .
$$

The quantizer $q_{\mathbb{U}}(\cdot)$ used in (14) gives the Euclidean projection of $-B^{T} P A x_{j} / \gamma$ on $\mathbb{U}$.

For $N>1$, calculating the horizon one laws in (14) requires significantly less computations than finding the minimizer in (7). However, it is by no means clear whether this simple control policy gives the same performance as would be provided by the quantized MPC formulation for arbitrary horizons $N \geq 2$. In the remainder of this work, we will investigate this question.

It is interesting to note that the horizon one law (14) can be interpreted as a greedy algorithm for the finite horizon optimization problem (7); see, e.g., [20]. Indeed, the paper examines performance of this particular greedy algorithm. 


\section{Optimality OF The Horizon One LaW}

In this section we develop necessary, and necessary and sufficient conditions for the horizon one controller to be equivalent to the horizon $N>1$ controller. We adopt the following notion:

Definition 1 (Initial Optimality): If for all $x_{0} \in \mathbb{R}^{n}$ there exist $u_{1}, \ldots, u_{N-1} \in \mathbb{U}$ such that the control sequence $\left(\kappa_{1}\left(x_{0}\right), u_{1}, \ldots, u_{N-1}\right)$ yields the optimal cost $V_{N}\left(x_{0}, \vec{u}\right)$, see (7), then we say that the horizon one law is initially optimal for horizon $N$.

It is worth emphasizing that, since the optimality notion adopted here is global, and plant and cost are time-invariant, initial optimality of the NCL does not depend on $\ell \in \mathbb{N}_{0}$.

A crucial feature of quantized MPC as presented in Section II is that the projection $q_{\widetilde{U}^{N}}(\cdot)$ used to characterize the optimizer $\vec{u}$ in (11) partitions $\mathbb{R}^{N}$ into Voronoi cells. This motivates us to adopt a geometric approach, which is based upon studying the sets

$$
\begin{aligned}
L_{k} & =\left\{\begin{array}{cc}
{[-\delta / 2, \delta / 2]} & \text { if } \quad k=0 \\
((k-1 / 2) \delta,(k+1 / 2) \delta] & \text { if } \quad k>0 \\
{[(k-1 / 2) \delta,(k+1 / 2) \delta)} & \text { if } \quad k<0
\end{array},\right. \\
Q_{k} & =\left\{z \in \mathbb{R}^{n} \mid-\frac{B^{T} P A z}{\gamma} \in L_{k}\right\}, k \in \mathbb{Z}, \\
M_{k, N}^{*} & =\left\{w \in \mathbb{R}^{N} \mid k \delta \in e_{1, N}^{T} H_{N}^{-1 / 2} q_{\widetilde{\mathbb{U}}^{N}}(w)\right\}, \\
C_{0, N}^{*} & =\left\{w \in \mathbb{R}^{N} \mid 0 \in q_{\widetilde{\mathbb{U}}^{N}}(w)\right\} .
\end{aligned}
$$

Lemma 1: Consider the sets in (15) and the control laws in (13) and (14). We then have:

1) The set $C_{0, N}^{*}$ is the Voronoi cell of the lattice

$$
\left\{\sum_{i=1}^{N} \delta k_{i} H_{N}^{1 / 2} e_{i, N} \mid k_{i} \in \mathbb{Z}\right\}
$$

that contains 0 ; see, e.g., [21]. The zero control sequence yields the optimal cost, iff $-H_{N}^{-1 / 2} F_{N} x_{0} \in C_{0, N}^{*}$. Moreover $C_{0, N}^{*}$ is the intersection of all the half-spaces

$$
\begin{array}{r}
J_{\vec{k}}=\left\{x \in \mathbb{R}^{N} \mid\left(2 x-H_{N}^{1 / 2} \vec{k}\right)^{T} H_{N}^{1 / 2} \vec{k} \leq 0\right\}, \\
\vec{k} \in \mathbb{U}^{N} \backslash\{0\} .
\end{array}
$$

2) For all $z \in \mathbb{R}^{n}$, it holds that $z \in \overline{Q_{k}}$, iff $k \delta \in \kappa_{1}(z)$.

3) For any $x_{0} \in \mathbb{R}^{n}$, we have $k \delta \in e_{1}^{T} \kappa_{N}\left(x_{0}\right)$, iff $-H_{N}^{-1 / 2} F_{N} x_{0} \in M_{k, N}^{*}$.

Proof: These properties follow directly from the definitions.

Some additional geometrical features of the sets introduced in (15) are established in the following lemma, whose proof is included in Appendix A.

Lemma 2: Consider the sets defined in (15). We then have:

1) $M_{k, N}^{*}=M_{0, N}^{*}+k \delta H_{N}^{1 / 2} e_{1, N}$.

2) $M_{k, N}^{*}$ is closed.

3) $M_{0, N}^{*}=C_{0, N}^{*}+\left\{\sum_{k=2}^{N} \delta m_{k} H_{N}^{1 / 2} e_{k, N} \mid m_{k} \in \mathbb{Z}\right\}$.

4) $\overline{\left(M_{k, N}^{*}\right)^{o}}=M_{k, N}^{*}$, i.e., the closure of the interior of $M_{k, N}^{*}$ equals $M_{k, N}^{*}$.

5) If $k \neq l$, then $\left(M_{k, N}^{*}\right)^{o} \cap M_{l, N}^{*}=\emptyset$.
The sets introduced above allow us to study initial optimality of the horizon one controller by using geometric tools. Our first result is stated in Theorem 1:

Theorem 1: If $N \geq 2$, then the horizon one controller is initially optimal for horizon $N$, iff

$$
-H_{N}^{-1 / 2} F_{N} Q_{k} \subset M_{k, N}^{*}, \quad \forall k \in \mathbb{Z} .
$$

Proof: The result is immediate from Lemma 1.

Theorem 1 establishes necessary and sufficient conditions for the horizon one controller to be initially optimal. Our results apply to any horizon $N>1$ and to LTI plants of any order $n \geq 1$. Unfortunately, condition (17) cannot be checked in practice. Thus, the remainder of the paper is devoted to establishing equivalent conditions which are checkable.

Before proceeding, we state Corollary 1.

Corollary 1: Suppose that the horizon one law is initially optimal for horizon $N$. Then, the horizon one law is initially optimal if, in (1), we replace $A$ by $-A$ and/or

$$
B \rightarrow T B \text { and } P \rightarrow T^{-T} P T^{-1} \text { and } Q \rightarrow T^{-T} Q T^{-1},
$$

where $T$ is invertible and commutes with $A$, i.e., it holds that $T A=A T$. Moreover, initial optimality is independent of the stepsize $\delta$ in (3).

Proof: If we replace $A$ by $-A$ in (1), then the new problem data becomes $H_{N}^{\prime}=S H_{N} S, F_{N}^{\prime}=S F_{N}, M_{k, n}^{\prime *}=$ $-S M_{k, n}^{*}$, and $Q_{k}^{\prime}=-Q_{k}$, where

$$
S=\left(\begin{array}{cccc}
1 & 0 & \ldots & 0 \\
0 & -1 & \ddots & \vdots \\
\vdots & \ddots & \ddots & 0 \\
0 & \ldots & 0 & (-1)^{N}
\end{array}\right) \in \mathbb{R}^{N \times N} .
$$

Likewise, if we apply the transformation as in (18), then the new data becomes $H_{N}^{\prime}=H_{N}, M_{k, N}^{* *}=M_{k, n}^{*}, Q_{k}^{\prime}=T Q_{k}$ and $F_{N}^{\prime}=T^{-1} F_{N}$. Replacing $\delta$ by $\delta^{\prime}$ only changes $M_{k, n}^{*}$ to $\left(\delta^{\prime} / \delta\right) M_{k, n}^{*}$ and $Q_{k}$ to $\left(\delta^{\prime} / \delta\right) Q_{k}$. The results now follow from Theorem 1.

The next two lemmas give necessary conditions for initial optimality of the horizon one law.

Lemma 3: If the horizon one law is initially optimal for horizon $N$, then it is necessary that

$$
H_{N}^{-1} F_{N}\left(\operatorname{Ker} B^{T} P A\right) \subseteq \operatorname{Span}\left(e_{2, N}, \ldots, e_{N, N}\right) .
$$

Moreover, if $F_{N}$ is onto, then we have equality in (19).

Proof: See Appendix B.

Lemma 4: Suppose that $B^{T} P A \neq 0$ and define

$$
w_{N}:=\frac{\gamma}{\left|A^{T} P B\right|^{2}} H_{N}^{-1 / 2} F_{N} A^{T} P B .
$$

Then $-H_{N}^{-1 / 2} F_{N} Q_{k}=H_{N}^{-1 / 2} F_{N}\left(\operatorname{Ker} B^{T} P A\right)+L_{k} w_{N}$, $\forall k \in \mathbb{Z}$. If, in addition, the horizon one law is initially optimal for horizon $N$, then

$$
e_{1, N}^{T} H_{N}^{-1 / 2} w_{N}=1 .
$$

Proof: See Appendix C.

We note that the necessary condition (20) given above is very easy to check, since it only involves elementary 
matrix calculations. As will become apparent in the following section, we can simplify the necessary and sufficient condition provided by Theorem 1 in some specific situations.

\section{Particular Cases}

In Section III we established conditions for the horizon one law to be initially optimal which hold in general situations. However, these conditions are not always easy to check. Thus, in the sequel, we will study several special cases, where the conditions simplify considerably.

\section{A. Situations where $F_{N}$ is onto}

Here we focus on instances where the horizon is less than or equal to the state dimension (i.e., where $N \leq n$ ) and, in particular, study situations where $F_{N}$ defined in (9) is onto. To derive our subsequent results, we let $q_{N}^{(i)}, 1 \leq i \leq N$ be the Euclidean projection of $H_{N}^{1 / 2} e_{i, N}$ on

$$
\begin{aligned}
H_{N}^{-1 / 2} \operatorname{Span}\left(e_{1, N}, \ldots, e_{i, N}\right) & \\
= & {\left[\operatorname{Span}\left(H_{N}^{1 / 2} e_{i+1, N}, \ldots, H_{N}^{1 / 2} e_{N, N}\right)\right]^{\perp} . }
\end{aligned}
$$

In particular, we have

$$
q_{N}^{(1)}=\frac{H_{N}^{-1 / 2} e_{1, N}}{e_{1, N}^{T} H_{N}^{-1} e_{1, N}} .
$$

We furthermore define

$$
S_{N}:=H_{N}^{1 / 2} \operatorname{Span}\left(e_{2, N}, \ldots, e_{N, N}\right)+L_{0} q_{N}^{(1)}
$$

and state the following lemmas:

Lemma 5: Suppose that the set $\left(b_{1}, \ldots, b_{N}\right)$ is some orthonormal basis of $\mathbb{R}^{N}$. If $\mu \in \mathbb{R}$, and $c>0$ are such that

$$
\left\{\mu b_{1}+\sum_{k=2}^{N} \lambda_{k} b_{k} \mid \lambda_{k} \in[-c, c]\right\} \subset \partial C_{0, N}^{*},
$$

then there exists some $p \in \mathbb{U}^{N}$ such that $2 \mu b_{1}=H_{N}^{1 / 2} p$.

Proof: See Appendix D.

Lemma 6: The set $\left(q_{N}^{(1)}, \ldots, q_{N}^{(N)}\right)$ is an orthogonal basis of $\mathbb{R}^{N}$, and

$$
S_{N}=\operatorname{Span}\left(q_{N}^{(2)}, \ldots, q_{N}^{(N)}\right)+L_{0} q_{N}^{(1)} .
$$

Proof: Define $c_{1}^{\prime}=H_{N}^{-1 / 2} e_{1, N}$ and $c_{1}=c_{1}^{\prime} /\left|c_{1}^{\prime}\right|$. If for some $1 \leq l<N$ the orthonormal basis $\left(c_{1}, \ldots, c_{l}\right)$ of $H_{N}^{-1 / 2} \operatorname{Span}\left(e_{1, N}, \ldots, e_{l, N}\right)$ is found, we let $c_{l+1}^{\prime}$ be the Euclidean projection of $H_{N}^{-1 / 2} e_{l+1, N}$ on $\left(\operatorname{Span}\left(c_{1}, \ldots, c_{l}\right)\right)^{\perp}=\operatorname{Span}\left(H_{N}^{1 / 2} e_{l+1, N}, \ldots, H_{N}^{1 / 2} e_{N, N}\right)$. Define $c_{l+1}:=c_{l+1}^{\prime} /\left|c_{l+1}^{\prime}\right|$. Then $\left(c_{1}, \ldots, c_{i}\right)$ is an orthonormal basis of $H_{N}^{-1 / 2} \operatorname{Span}\left(e_{1, N}, \ldots, e_{i, N}\right)$ for all $1 \leq i \leq N$, and

$$
c_{l}^{T} H_{N}^{1 / 2} e_{i, N}=0, \quad \text { if } 1 \leq l<i \leq N .
$$

Thus,

$$
q_{N}^{(i)}=\sum_{k=1}^{i}\left(e_{i, N} H_{N}^{1 / 2} c_{k}\right) c_{k}=\left(e_{i, N}^{T} H_{N}^{1 / 2} c_{i}\right) c_{i} .
$$

Since $e_{i, N}^{T} H_{N}^{1 / 2} c_{i} \neq 0$, it follows that $\left(q_{N}^{(1)}, \ldots, q_{N}^{(N)}\right)$ is an orthogonal basis. The remainder of the proof is straightforward.

Lemma 7: Let $F_{N}$ be onto and $B^{T} P A \neq 0$. Then, necessary conditions for initial optimality of the horizon one law are:

1) $\left(M_{k, N}^{*}\right)^{0} \subset-H_{N}^{1 / 2} F_{N} Q_{k} \subset M_{k, N}^{*}$, for all $k \in \mathbb{Z}$.

2) $S_{N}=M_{0, N}^{*}$.

Proof: 1) Let $z \in\left(M_{k, N}^{*}\right)^{0}$. Since $F_{N}$ is onto, we have $\mathbb{R}^{N}=\bigcup_{l \in \mathbb{Z}}-H_{N}^{-1 / 2} F_{N} Q_{l}$. Thus, there exists some $l \in \mathbb{Z}$ and some $w \in Q_{l}$ such that $z=-H_{N}^{-1 / 2} F_{N} w$. By (11) we have $l \delta \in \kappa_{1}(w)=e_{1, N}^{T} H_{N}^{-1 / 2} q_{\widetilde{U}^{N}}(z)$, so that $z \in M_{l, N}^{*}$. Lemma 2 then gives $k=l$, which shows 1 ).

2) Lemmas 2, 3, 4 and 6 establish that

$$
\begin{aligned}
M_{0, N}^{*} & =\overline{\left(M_{0, N}^{*}\right)^{0}}=\overline{-H_{N}^{-1 / 2} F_{N} Q_{0}} \\
& =H_{N}^{1 / 2} \operatorname{Span}\left(e_{2, N}, \ldots, e_{N, N}\right)+L_{0} w_{N} .
\end{aligned}
$$

We, thus, obtain:

$$
\begin{aligned}
v \in M_{0, N}^{*} \Leftrightarrow & \exists \mu \in L_{0}: \\
& v-\mu w_{N} \in H_{N}^{1 / 2} \operatorname{Span}\left(e_{2, N}, \ldots, e_{N, N}\right) \\
= & {\left[\operatorname{Span}\left(H_{N}^{-1 / 2} e_{1, N}\right)\right]^{\perp} } \\
\Leftrightarrow & \exists \mu \in L_{0}: e_{1, N}^{T} H_{N}^{-1 / 2}\left(v-\mu w_{N}\right)=0 \\
\Leftrightarrow & \exists \mu \in L_{0}: e_{1, N}^{T} H_{N}^{-1 / 2} v=\mu \\
\Leftrightarrow & \exists \mu \in L_{0}, \exists \lambda_{2}, \ldots, \lambda_{N} \in \mathbb{R}: \\
& v=\mu q_{N}^{(1)}+\sum_{k=2}^{N} \lambda_{k} H_{N}^{1 / 2} e_{k, N} \Leftrightarrow v \in S_{N} .
\end{aligned}
$$

This proves the result.

The above technical results allow us to state Theorem 2. It gives necessary and sufficient conditions for the horizon one law to be initially optimal for situations where $F_{N}$ is onto.

Theorem 2: Let $N \geq 2$, let $F_{N}$ be onto and $B^{T} P A \neq$ 0 . Then, the necessary and sufficient conditions for $\kappa_{1}$ to be initially optimal for horizon $N$ are that the following 3 conditions hold:

1) $q_{N}^{(1)} \in \mathbb{Z}^{N}$,

2) $F_{N}\left(K \operatorname{Ker} B^{T} P A\right)=H_{N} \operatorname{Span}\left(e_{2, N}, \ldots, e_{N, N}\right)$,

3) $\gamma e_{1, N}^{T} H_{N}^{-1} F_{N} A^{T} P B=\left|A^{T} P B\right|^{2}$.

Proof: See Appendix E.

We note that, using Cramer's rule, 1) in Theorem 2 is equivalent to, see (21), $\operatorname{det} H_{N}^{(1, l)} / \operatorname{det} H_{N}^{(1,1)} \in \mathbb{Z}, \forall l \in\{1, \ldots N\}$, where $H_{N}^{(1, l)}$ is $H_{N}$ after omitting the first row and the $l$-th column; in 2), the equality can be replaced by $\subset$; 3 ) means that $e_{1, N}^{T} H_{N}^{-1 / 2} w_{N}=1$.

\section{B. Plant Models where $B$ belongs to the image of $A$}

Throughout this section, we assume that, in the model (1), we have $B \in \operatorname{Im} A$. This is actually a common case since $A$ is always nonsingular when the model arises from sampling a continuous-time system. Our first result, namely Lemma 8, establishes necessary and sufficient conditions for the special case where $R=0$. 
Lemma 8: Let $R=0$ and $B=A \mu$ for some $\mu \in \mathbb{R}^{n}$. Then the horizon one law is initially optimal for horizon $N$, iff $-H_{N}^{-1 / 2} F_{N} Q_{0} \subset M_{0, N}^{*}$.

Proof: See Appendix F.

Next, we return to the general case $R \neq 0$. Theorem 1 establishes that the horizon one law is initially optimal, iff $-H_{N}^{-1 / 2} F_{N} Q_{k} \subset M_{k, N}^{*}$ for all $k \in \mathbb{Z}$. Since, by Lemma 2, we have

$$
\begin{aligned}
& H_{N}^{-1 / 2} M_{k, N}^{*}=H_{N}^{-1 / 2} C_{0, N}^{*} \\
& \quad+\delta\left\{\left(k, m_{2}, \ldots, m_{N}\right)^{T} \mid m_{2}, \ldots, m_{N} \in \mathbb{Z}\right\},
\end{aligned}
$$

where $M_{k, N}^{*}$ is closed, it turns out that the condition in Theorem 1 is equivalent to the requirement:

$$
\begin{aligned}
& \forall k \in \mathbb{Z}, \forall w \in Q_{k}^{0}, \exists m_{2}, \ldots, m_{N} \in \mathbb{Z}: \\
& \quad-H_{N}^{-1} F_{N} w-\left(k, m_{2}, \ldots, m_{N}\right)^{T} \in H_{N}^{-1 / 2} C_{0, N}^{*} .
\end{aligned}
$$

On the other hand, $C_{0, N}^{*}=\bigcap_{\vec{k} \in \mathbb{U}^{N}} J_{\vec{k}}$, where $J_{\vec{k}}$ is as in (16). This implies that $x \in H_{N}^{-1 / 2} C_{0, N}^{*}$, iff $x^{T} H_{N} \vec{k} \leq \vec{k}^{T} H_{N} \vec{k} / 2$ for all $\vec{k} \in \mathbb{U}^{N}$. Thus, (23) is equivalent to

$$
\begin{aligned}
\forall k \in \mathbb{Z}, \forall w \in Q_{k}^{0}, \exists m_{2}, \ldots, m_{N} \in \mathbb{Z}, \forall \vec{k} \in \mathbb{U}^{N}: \\
\qquad \vec{k}^{T}\left[F_{N} w+\delta H_{N}\left(\begin{array}{c}
k \\
m_{2} \\
\vdots \\
m_{N}
\end{array}\right)\right] \leq \frac{1}{2} \vec{k}^{T} H_{N} \vec{k} .
\end{aligned}
$$

Our working assumption in this section is that $B \in \operatorname{Im} A$. Hence, Lemma 8 gives that (24) is equivalent to the condition:

$$
\begin{aligned}
& \forall k \in \mathbb{Z}, \forall w \in Q_{0}^{0} / \delta, \exists m \in \mathbb{Z}^{N-1}, \forall l \in \mathbb{Z}^{N}: \\
& l^{T} H_{N}\left[H_{N}^{-1} F_{N}\left(w-\frac{k \gamma \mu}{B^{T} P B}\right)+\left(\begin{array}{c}
k \\
m
\end{array}\right)\right] \leq \frac{l^{T} H_{N} l}{2},
\end{aligned}
$$

i.e.,

$$
\begin{array}{r}
l^{T} H_{N}\left[H_{N}^{-1} F_{N} w-\frac{k R}{B^{T} P B}\left(E_{N}-\gamma H_{N}^{-1}\right) e_{1, N}+\left(\begin{array}{c}
0 \\
m
\end{array}\right)\right] \\
\leq \frac{1}{2} l^{T} H_{N} l, \quad(26)
\end{array}
$$

where $Q_{0}^{0} / \delta=\left\{z \in \mathbb{R}^{n} \mid B^{T} P A z / \gamma \in(-1 / 2,1 / 2)\right\}$.

Expression (26) has an interesting geometric interpretation: The equation $x^{T} H_{N} x=d^{2}$ represents an ellipsoid in $\mathbb{R}^{N}$ with half axis $d v_{i} / \sqrt{d_{i}}, 1 \leq i \leq N$, where $H_{N}=\mathcal{O D O}^{T}$ is the singular value decomposition of $H_{N}$, the orthogonal matrix $\mathcal{O}$ consisting of the eigenvectors of $H_{N}$ as columns, and the diagonal matrix $D$ has the eigenvalues $d_{1}, \ldots, d_{N}$ of $H_{N}$ in its diagonal. The inequality $l^{T} H_{N} x \leq l^{T} H_{N} l / 2$ for all $l \in \mathbb{Z}^{N}$ is equivalent to the function $t \mapsto(t-x)^{T} H_{N}(t-x)-x^{T} H_{N} x$ taking no negative values on $\mathbb{Z}^{N}$, i.e., the ellipsoid with center $x$ and half axis $\sqrt{x^{T} H_{N} x / d_{i}} v_{i}, 1 \leq i \leq N$ containing no point of $\mathbb{Z}^{N}$ in its interior.

Further insight into condition (26) is provided by the following lemma:
Lemma 9: Define $G_{x}: \mathbb{Z}^{N-1} \rightarrow \mathbb{R}$ by

$$
G_{x}(k):=\left(x+\left(\begin{array}{l}
0 \\
k
\end{array}\right)\right)^{T} H_{N}\left(x+\left(\begin{array}{l}
0 \\
k
\end{array}\right)\right),
$$

and the constrained minimizer

$$
r_{x}:=\min _{k \in \mathbb{Z}^{N-1}} G_{x}(k) .
$$

Furthermore, let $x \in \mathbb{R}^{N}$, and assume that there exists some $m \in \mathbb{Z}^{N-1}$ such that $l^{T} H_{N}\left[x+\left(\begin{array}{c}0 \\ m\end{array}\right)\right] \leq \frac{1}{2} l^{T} H_{N} l$ for all $l \in \mathbb{Z}^{N}$

Then, $l^{T} H_{N}\left[x+\left(\begin{array}{c}0 \\ m_{x}\end{array}\right)\right] \leq \frac{1}{2} l^{T} H_{N} l, \forall l \in \mathbb{Z}^{N}$, where $m_{x} \in \mathbb{Z}^{N-1}$ is any minimizer of $G_{x}$.

Proof: Consider an ellipsoid $E$ with center $x+\left(\begin{array}{c}0 \\ m_{x}\end{array}\right)$ and half axis $\sqrt{r_{x} / d_{i}} v_{i}, 1 \leq i \leq N$. Now, assume that $E$ contains some point, $k \in \mathbb{Z}^{N}$, in its interior. By assumption, the ellipsoid $\widetilde{E}$ with center $x+\left(\begin{array}{c}0 \\ m\end{array}\right)$ and half axis $\sqrt{G_{x}(m) / d_{i}} v_{i}, 1 \leq i \leq N$, does not contain a point of $\mathbb{Z}^{N}$ in its interior. However, we have $E+\left(\begin{array}{c}0 \\ m-m_{x}\end{array}\right) \subset \widetilde{E}$, which gives $k+\left(\begin{array}{c}0 \\ m-m_{x}\end{array}\right) \subset \widetilde{E}^{0}$, a contradiction.

The understanding gained by the geometric considerations described above allows us to state the following necessary condition for initial optimality of the horizon one law:

Corollary 2: Let $B \in \operatorname{Im} A$. Then, a necessary condition for initial optimality of $\kappa_{1}$ is:

$$
R\left(1-\gamma e_{1, N}^{T} H_{N}^{-1} e_{1, N}\right)=0,
$$

or, in other words, $R\left(\operatorname{det} H_{N}-\gamma \operatorname{det} H_{N-1}\right)=0$.

Proof: See Appendix G.

Note that the function $R \mapsto \operatorname{det} H_{N}-\gamma \operatorname{det} H_{N-1}$ is a polynomial of degree at most $N-1$. Hence, checking (27) is equivalent to checking the roots of an $N-1$ degree polynomial. (We recall that the degree of the zero polynomial is $-\infty$. Moreover, $H_{1}=\gamma=B^{T} P B+R \in(0,+\infty)$.)

\section{First order Plant Models and Horizon $N=2$}

We will next show how to use the results presented so far in the special case of state dimension $n=1$, horizon $N=2$ and control weighting $R=0$. This complements the results in [14], where only sufficient conditions were given for initial optimality.

With $n=1, N=2$ and $R=0$, we have

$$
\begin{aligned}
F_{N}=\left(\begin{array}{c}
B A\left(Q+A^{2} P\right) \\
B P A^{2}
\end{array}\right), \quad H_{N} & =\left(\begin{array}{cc}
\alpha & \beta \\
\beta & \gamma
\end{array}\right), \\
w_{N} & =(B / A) H_{N}^{-1 / 2} F_{N},
\end{aligned}
$$

with $\alpha=B^{2}\left(Q+P A^{2}\right), \beta=B^{2} A P, \gamma=B^{2} P$.

Direct calculation gives that $w_{N}=e_{1, N}$, that is, the necessary condition of Lemma 4 is automatically satisfied. Since $-H_{N}^{-1 / 2} F_{N} Q_{0}=(-1 / 2,1 / 2)(B / A) H_{N}^{-1 / 2} F_{N}$, we see that the horizon one law is initially optimal, iff 
$(-1 / 2,1 / 2)(B / A) H_{N}^{-1 / 2} F_{N} \subset M_{0, N}^{*}$, that is, for all $|t| \leq$ $1 / 2$ there is some $k \in \mathbb{Z}$ such that, for all $l, m \in \mathbb{Z}$,

$\left|t \frac{B}{A} H_{N}^{-1 / 2} F_{N}-H_{N}^{1 / 2}\left(\begin{array}{l}0 \\ k\end{array}\right)\right| \leq\left|t \frac{B}{A} H_{N}^{-1 / 2} F_{N}-H_{N}^{1 / 2}\left(\begin{array}{c}l \\ m\end{array}\right)\right|$,

in other words, iff for all $|t| \leq 1 / 2$ the function $x \mapsto$ $x^{T} H_{N} x-2 t \frac{B}{A} x^{T} F_{N}$ takes its minimum on $\mathbb{Z}^{2}$ in the set $\{0\} \times \mathbb{Z}$. Letting $z=2 t(B / A) F_{N}$, we see that

$$
\begin{gathered}
x^{T} H_{N} x-x^{T} z=\left(x-\frac{1}{2} H_{N}^{-1} z\right)^{T} H_{N}\left(x-\frac{1}{2} H_{N}^{-1} z\right) \\
-\frac{z^{T} H_{N}^{-1} z}{4}=\left(x-\left(\begin{array}{c}
t \\
0
\end{array}\right)\right)^{T} H_{N}\left(x-\left(\begin{array}{c}
t \\
0
\end{array}\right)\right)-\frac{z^{T} H_{N}^{-1} z}{4} .
\end{gathered}
$$

The ellipse $\left(x-\left(\begin{array}{l}t \\ 0\end{array}\right)\right)^{T} H_{N}\left(x-\left(\begin{array}{l}t \\ 0\end{array}\right)\right)=d^{2}$ has the vertical line $x_{1}=k, k \in \mathbb{Z}$, as tangent, iff $d^{2}=(k-t)^{2}(\alpha \gamma-$ $\left.\beta^{2}\right) / \gamma$. In this case, it intersects the vertical line $x_{1}=k$ at the point $(k, \gamma(t-k) / \gamma)^{T}$. As a consequence, the function

$$
g(x):=\left(x-\left(\begin{array}{l}
t \\
0
\end{array}\right)\right)^{T} H_{N}\left(x-\left(\begin{array}{l}
t \\
0
\end{array}\right)\right)
$$

takes its minimum on $\{k\} \times \mathbb{Z}$ in $\left(k,\left\lfloor r_{k}\right\rfloor\right)^{T}$, where

$$
r_{k}:=1 / 2+\beta(t-k) / \gamma=1 / 2+A(t-k) .
$$

The requirement that $g$ takes its minimum on $\mathbb{Z}^{2}$ in $\{0\} \times \mathbb{Z}$ is equivalent to demanding that

$$
\left(\begin{array}{c}
-t \\
\left\lfloor r_{0}\right\rfloor
\end{array}\right)^{T} H_{N}\left(\begin{array}{c}
-t \\
\left.r_{0}\right\rfloor
\end{array}\right) \leq\left(\begin{array}{c}
k-t \\
\left\lfloor r_{k}\right\rfloor
\end{array}\right)^{T} H_{N}\left(\begin{array}{c}
k-t \\
\left\lfloor r_{k}\right\rfloor
\end{array}\right), \quad \forall k \in \mathbb{Z}
$$

We conclude that the horizon one law is initially optimal, iff $\alpha t^{2}-2 \beta\left\lfloor r_{0}\right\rfloor t+\gamma\left\lfloor r_{0}\right\rfloor^{2} \leq \alpha(k-t)^{2}-2 \beta\left\lfloor r_{k}\right\rfloor(t-k)+\gamma\left\lfloor r_{k}\right\rfloor^{2}$,

for all $|t| \leq 1 / 2$, for all $k \in \mathbb{Z}$. Since $\left(\alpha \gamma-\beta^{2}\right) / \gamma^{2}=Q / P$ and $\beta / \gamma=A$, the inequality in (28) can be re-written as

$$
\begin{aligned}
\mathcal{K}_{k}(t):= & (t-k)^{2}+\frac{P}{Q}\left(\left\lfloor r_{k}\right\rfloor-A(t-k)\right)^{2}-t^{2} \\
& -\frac{P}{Q}\left(\left\lfloor r_{0}\right\rfloor-A t\right)^{2} \geq 0, \quad \forall|t| \leq 1 / 2, \forall k \in \mathbb{Z} .
\end{aligned}
$$

We will next further elucidate this condition and, thus, obtain necessary and sufficient conditions on the horizon one controller to be optimal for horizon 2.

By Corollary 1, we only need to examine $A>0$. We note that $\mathcal{K}_{k}$ is twice differentiable from the right everywhere,

$\frac{d^{+} \mathcal{K}_{k}}{d t}(t)=2 A \frac{P}{Q}\left(\left\lfloor r_{0}\right\rfloor-A t\right)-2 A \frac{P}{Q}\left(\left\lfloor r_{k}\right\rfloor-A(t-k)\right)-2 k$

and

$$
\frac{d^{+}}{d t} \frac{d^{+} \mathcal{K}_{k}}{d t}(t)=0
$$

The points of discontinuity of $\frac{d^{+} \mathcal{K}_{k}}{d t}$ on $(-1 / 2,1 / 2)$ are contained in $\mathcal{A}_{k} \cup \mathcal{B}_{k}$ :

$$
\begin{aligned}
& \mathcal{A}_{k}:=\left\{t \in(-1 / 2,1 / 2] \mid r_{0} \in \mathbb{Z} \text { and } r_{k} \notin \mathbb{Z}\right\} \\
& \mathcal{B}_{k}:=\left\{t \in(-1 / 2,1 / 2) \mid r_{0} \notin \mathbb{Z} \text { and } r_{k} \in \mathbb{Z}\right\} .
\end{aligned}
$$

Therefore, on any interval $I \subset(-1 / 2,1 / 2)$ that does not intersect the finite set $\mathcal{A}_{k} \cup \mathcal{B}_{k}$, the function $\mathcal{K}_{k}$ is affinelinear. Thus, $\mathcal{K}_{k} \geq 0$ on $[-1 / 2,1 / 2]$, iff $\mathcal{K}_{k}(-1 / 2) \geq 0$, $\mathcal{K}_{k}(1 / 2) \geq 0$ and $\mathcal{K}_{k}(v) \geq 0$, for all $v \in \mathcal{A}_{k} \cup \mathcal{B}_{k}$.

Since $(\lfloor 1 / 2+A / 2\rfloor-A / 2)^{2}=(\lfloor 1 / 2-A / 2\rfloor+A / 2)^{2}$, we have $\mathcal{K}_{k+1}(1 / 2)=\mathcal{K}_{k}(-1 / 2)$ for all $k \in \mathbb{Z}$. Furthermore, it is easy to see that $\mathcal{K}_{k}(t) \geq 0$, for all $|t| \leq 1 / 2$ and all $t \in \mathcal{B}_{k}$. Thus, $\mathcal{K}_{k} \geq 0$ on $[-1 / 2,1 / 2]$ for all $k \in \mathbb{Z}$, iff $\mathcal{K}_{k}(v) \geq 0$, for all $v \in \mathcal{A}_{k} \cup\{-1 / 2\}$ and all $k \in \mathbb{Z}$.

First, we consider $A>1$ and $A \notin \mathbb{N}$. Then,

$$
\max \mathcal{A}_{1}=(\lfloor A / 2+1 / 2\rfloor-1 / 2) / A,
$$

and $\mathcal{K}_{1}\left(\max \mathcal{A}_{1}\right) \geq 0$ is equivalent to

$$
\frac{Q}{P} \geq \frac{A(A-\lfloor A\rfloor)(\lfloor A\rfloor+1-A)}{A-2\left\lfloor\frac{A+1}{2}\right\rfloor+1} .
$$

Conversely, using right-hand derivative techniques, it can be shown that (30) implies $\mathcal{K}_{k}(t) \geq 0$ for all $t \in \mathcal{A}_{k}$ and all $k$. Moreover, it can be shown that $\mathcal{K}_{k}(-1 / 2) \geq 0$ for all $k \in \mathbb{Z}$, iff

$$
\frac{Q}{P} \geq \frac{(r-\lfloor r\rfloor)(\lfloor r\rfloor+1-r)}{r^{2}\lfloor r\rfloor(\lfloor r\rfloor+1)},
$$

where $1 / r=A-\lfloor A\rfloor$, if $\lfloor A\rfloor$ is even, and $1 / r=\lfloor A\rfloor+1-A$, otherwise. For $A>1, A \notin \mathbb{N}$ inequality (30) implies (31) and, therefore, (30) is the necessary and sufficient condition.

We will next consider $A \in \mathbb{N}^{+}$. Then, $\mathcal{A}_{k}=\emptyset$, and it is easy to see that $\mathcal{K}_{k}(-1 / 2) \geq 0$. Thus, with $A \in \mathbb{N}^{+}$, the quantized horizon one controller is always initially optimal for horizon 2.

Finally, we consider $0<A<1$. Then, again $\mathcal{A}_{k}=\emptyset$, and (31) amounts to a necessary and sufficient condition for initial optimality.

The preceding analysis leads to the conclusion that, for plants of order $n=1$ and horizon $N=2$, the inequalities (30) and (31) give necessary and sufficient conditions for initial optimality, when $A>1$ and $A \in(0,1)$, respectively. It is interesting to note that, even in this special case, the conditions are non-trivial.

\section{Near Optimality}

A difficulty with the optimality conditions is that they hold for very special choices of the problem data $(A, B, P, Q, R)$. We view these variables as being part of the problem description and not as design choices. This leads to the question: What if the necessary and sufficient conditions are "almost" satisfied? Will the horizon one law be "almost optimal"? This is addressed in the sequel. To state our result, in Theorem 3, we first give the following two lemmas:

Lemma 10: Let $z, w \in \mathbb{R}^{n}$, let $0 \in \mathbb{V} \subset \mathbb{R}^{n}$ be a closed set, and let $H, L$ be two invertible $n \times n$ matrices. Then

$$
\begin{aligned}
|H t-z|^{2} & -|L s-w|^{2} \\
& \leq\left(2|| H-L||\left|L^{-1}\right||| w|+| w-z \mid\right)(|w|+|z|)
\end{aligned}
$$

for all $t \in H^{-1} q_{H \mathbb{V}}(z)$ and all $s \in L^{-1} q_{L \mathbb{V}}(w)$. 
Proof: We have $|H t-z| \leq|H q-z|$ for all $q \in \mathbb{V}$. Thus, $|H t-z| \leq|z|$ and

$$
\begin{array}{r}
|H t-z| \leq|H s-z|=|(H-L) s+w-z+L s-w| \\
\leq|L s-w|+|| H-L|||s|+|w-z| .
\end{array}
$$

Since $|L s-w| \leq|w|$, we also have

$$
|s|=\left|L^{-1}(L s-w)+L^{-1} w\right| \leq 2\left\|L^{-1}\right\||w| .
$$

This gives

$$
\begin{aligned}
& |H t-z|^{2}-|L s-w|^{2} \\
& \quad \leq(|| H-L|||s|+|w-z|)(|H t-z|+|L s-w|),
\end{aligned}
$$

which proves the result.

Lemma 11: Let $x_{0}, c \in \mathbb{R}^{n}$ with $c \neq 0$ and $k \in \mathbb{Z}$. Then the problem to minimize $\alpha\left(\widehat{x}_{0}\right):=\left|x_{0}-\widehat{x}_{0}\right|$ subject to $c^{T} \widehat{x}_{0} \in \bar{L}_{k}$ has a unique solution $\hat{x}_{0}$, and

$$
\alpha_{\text {min }}= \begin{cases}0 & \text { if }\left|c^{T} x_{0}-k \delta\right| \leq \delta / 2, \\ \frac{\left|c^{T} x_{0}-k \delta\right|-\delta / 2}{|c|} & \text { otherwise. }\end{cases}
$$

Proof: Define $f(x)=c^{T} x$. Then

$$
f^{-1}\left(\overline{L_{k}}\right)=\left[-\frac{\delta}{2|c|}, \frac{\delta}{2|c|}\right] \frac{c}{|c|}+\frac{k \delta c}{|c|^{2}}+\operatorname{Ker} f
$$

is a strip in $\mathbb{R}^{n}$ of width $\delta /|c| . c$ is perpendicular on $\operatorname{Ker} f$, and the optimal $\widehat{x}_{0}$ is the Euclidean projection of $x_{0}$ on this strip. Since the distance between $x_{0}$ and $k \delta c /\left(|c|^{2}\right)+\operatorname{Ker} f$ is $\left|c^{T} x_{0}-k \delta\right| /(|c|)$, we see that $\alpha_{\min }=0$, if this distance is less or equal to $\delta /(2|c|)$, whereas $\alpha_{\min }=\left(2\left|c^{T} x_{0}-k \delta\right|-\delta\right) /(2|c|)$ otherwise.

To analyze sub-optimality of the horizon one controller, we will investigate the optimization problem with data $\left(A^{\prime}, B^{\prime}, P^{\prime}, Q^{\prime}, R^{\prime}\right)$, see (4), henceforth denoted by $V_{N}^{\prime}\left(x_{0}, \cdot\right)$. For this data, we will denote the optimizer by:

$$
\bar{u}^{\prime} \in \arg \min _{\vec{u}^{\prime} \in \mathbb{U}^{N}} V_{N}^{\prime}\left(x_{0}, \vec{u}^{\prime}\right) .
$$

The associated horizon one controller satisfies:

$$
\kappa_{1}^{\prime}\left(x_{0}\right)=q_{\mathbb{U}}\left(-B^{\prime T} P^{\prime} A^{\prime} x_{0} / \gamma,\right), \quad \gamma^{\prime}:=B^{\prime T} P^{\prime} B^{\prime}+R^{\prime} .
$$

Given the above, we will study the degree of sub-optimality of the control sequence

$$
u^{\prime}=\left(\kappa_{1}^{\prime}\left(x_{0}\right), u_{1}^{\prime}, \ldots, u_{N-1}^{\prime}\right),
$$

where $\left(u_{1}^{\prime}, \ldots, u_{N-1}^{\prime}\right)$ is some minimizer of $V_{N-1}^{\prime}\left(A^{\prime} x_{0}+\right.$ $\left.B^{\prime} u_{0}^{\prime}, \cdot\right)$ in $\mathbb{U}^{N-1}$.

Theorem 3: Consider some optimizer $\bar{u}^{\prime}$, see (32), and the sequence $u^{\prime}$ introduced in (34). Suppose that $\kappa_{1}\left(x_{0}\right)$ is initially optimal for the problem with data $(A, B, P, Q, R)$, where $B^{T} P A \neq 0$.
The difference in achieved cost is then bounded by:

$$
\begin{gathered}
V_{N}^{\prime}\left(x_{0}, u^{\prime}\right)-V_{N}^{\prime}\left(x_{0}, \bar{u}^{\prime}\right) \leq\left|x_{0}\right|^{2} \varepsilon_{Q}+\alpha\|Q\|\left(\left|x_{0}\right|+\mu\right) \\
+\left[2 d_{1}\left\|H_{N-1}^{\prime 1 / 2}-H_{N-1}^{1 / 2}\right\| \cdot\left\|H_{N-1}^{-1 / 2}\right\| \cdot\left\|T_{N-1}\right\|\right. \\
\left.+d_{2} \varepsilon_{T_{N-1}}+\varepsilon_{1}\left\|T_{N-1}\right\|\right] \cdot\left[d_{1}\left\|T_{N-1}\right\|+d_{2}\left\|T_{N-1}^{\prime}\right\|\right] \\
+\left(\varepsilon_{L_{N-1}}+\varepsilon_{Q}+\varepsilon_{W_{N-1}}\right) d_{1}^{2}+\beta_{N-1}^{\prime}\left(d_{1}+d_{2}\right) \varepsilon_{1} \\
+\mu^{2}\left(\varepsilon_{L_{N}}+\varepsilon_{Q}\right)+4 \mu \varepsilon_{F_{N}}\left|T_{N}^{\prime} x_{0}\right| \cdot\left\|H_{N}^{\prime-1 / 2}\right\| \\
+4 \varepsilon_{H_{N}}\left|T_{N}^{\prime} x_{0}\right|^{2}\left\|H_{N}^{\prime-1 / 2}\right\|^{2}+\alpha\left(\left|x_{0}\right|+\mu\right)\left(\beta_{N}^{\prime}+\left\|T_{N}^{\prime}\right\|^{2}\right) .
\end{gathered}
$$

The constants appearing are as follows:

$$
\alpha=\left\{\begin{array}{c}
0 \\
\frac{\left|c^{T} x_{0}-u_{0}^{\prime}\right|-\delta / 2}{|c|}
\end{array}\right.
$$

$$
\begin{array}{cc}
\text { if } & \left|c^{T} x_{0}-u_{0}^{\prime}\right| \leq \delta / 2 \\
\text { otherwise } & \\
& \leq \frac{\left|c-c^{\prime}\right|}{|c|}\left|x_{0}\right|,
\end{array}
$$

where

$$
\begin{aligned}
c^{T} & =-B^{T} P A / \gamma, c^{T}=-B^{\prime T} P^{\prime} A^{\prime} / \gamma^{\prime}, T_{N}=H_{N}^{-1 / 2} F_{N}, \\
\mu & =\left|x_{0}\right|+\alpha, d_{1}=\alpha\|A\|+\left|A x_{0}+B u_{0}^{\prime}\right|, \\
d_{2} & =\left|A^{\prime} x_{0}+B^{\prime} u_{0}^{\prime}\right|, \varepsilon_{T}=\left\|T_{N}^{\prime}-T_{N}\right\|, \\
\varepsilon_{1} & =\alpha\|A\|+\left\|A-A^{\prime}\right\|\left|x_{0}\right|+\left|B^{\prime}-B \| u_{0}^{\prime}\right|, \\
L_{N} & =\Lambda^{T} \widehat{Q}_{N} \Lambda_{N}, \varepsilon_{L_{N}}=\left\|L_{N}^{\prime}-L_{N}\right\|, W_{N}=T_{N}^{T} T_{N}, \\
\varepsilon_{W_{N}} & =\left\|W_{N}^{\prime}-W_{N}\right\|, \beta_{N}^{\prime}=\left\|L_{N}^{\prime}+Q^{\prime}\right\|+\left\|W_{N}^{\prime}\right\|, \\
\varepsilon_{F_{N}} & =\left\|F_{N}^{\prime}-F_{N}\right\|, \varepsilon_{H_{N}}=\left\|H_{N}^{\prime}-H_{N}\right\|, \varepsilon_{Q}=\left\|Q^{\prime}-Q\right\| .
\end{aligned}
$$

Proof: See Appendix H.

The significance of the above result is that it gives a bound on the degradation in performance when the system is perturbed from the nominal one. Moreover, the inequality is sharp in the sense that the difference in performance decreases to zero as the perturbed system approaches the nominal system.

Remark 1: If we use the matrix norm

$$
\|A\|_{2}=\max _{|x|=1}|A x|,
$$

then some of the constants used in Theorem 3 can be upper bounded as follows: Firstly, for all positive definite matrices $M, K$, we have $\left\|M^{1 / 2}\right\|_{2}=\sqrt{\sigma_{\max }(M)}$ and $\left\|M^{-1 / 2}\right\|_{2}=$ $1 / \sqrt{\sigma_{\min }(M)}$, where $\sigma_{\max }(M)$ and $\sigma_{\min }(M)$ denote the biggest and smallest eigenvalue of $M$, respectively. It furthermore can be shown that

$$
\begin{aligned}
& \left\|M^{1 / 2}-K^{1 / 2}\right\|_{2} \\
& \quad \leq \frac{1}{\sqrt{2}}\|M-K\|_{2} \cdot\left(\min \left\{\sigma_{\min }(M), \sigma_{\min }(K)\right\}\right)^{-1 / 2},
\end{aligned}
$$

which can be used to bound $\left\|H_{N-1}^{\prime 1 / 2}-H_{N-1}^{1 / 2}\right\|_{2}$.

To bound $\varepsilon_{W_{N}}$, one can use the fact that for any matrices $X, Y$ of compatible dimensions, it holds that

$$
\begin{aligned}
& \left\|X^{T} K^{-1} X-Y^{T} M^{-1} Y\right\|_{2} \\
& =\|(X-Y)^{T} K^{-1}(X-Y)+(X-Y)^{T} K^{-1} Y \\
& \quad+Y^{T} K^{-1}(X-Y)+Y^{T} K^{-1}(M-K) M^{-1} Y \|_{2} \\
& \leq\|X-Y\|_{2}^{2}\left\|K^{-1}\right\|_{2}+2\|X-Y\|_{2}\|Y\|_{2}\left\|K^{-1}\right\|_{2} \\
& \quad+\|Y\|_{2}^{2}\left\|K^{-1}\right\|_{2}\left\|M^{-1}\right\|_{2}\|M-K\|_{2},
\end{aligned}
$$


which shows:

$\varepsilon_{W_{N}} \leq\left\|H_{N}^{\prime-1}\right\|_{2}\left[\varepsilon_{F_{N}}^{2}+2 \varepsilon_{F_{N}}\left\|F_{N}\right\|_{2}+\left\|F_{N}\right\|_{2}^{2}\left\|H_{N}^{-1}\right\|_{2} \varepsilon_{H_{N}}\right]$.

Finally, the bound

$$
\left|T_{N}^{\prime} x_{0}\right|\left\|H_{N}^{\prime-1 / 2}\right\|_{2} \leq \frac{\left\|F_{N}^{\prime}\right\|_{2}\left|x_{0}\right|}{\sigma_{\min }\left(H_{N}^{\prime}\right)}
$$

is useful in practice.

\section{EXAMPLES}

Here, we present examples, which illustrate the ideas presented in this paper.

\section{A. $B \in \operatorname{Im} A$}

For the case horizon $N=2$ and $R \neq 0$ a necessary condition for the horizon one law to be initially optimal is:

$$
\left(R+B^{T} P B\right) B^{T}\left(Q-P+A^{T} P A\right) B=\left(B^{T} P A B\right)^{2} .
$$

This follows from Corollary 2. The above condition is satisfied if $P$ is chosen to satisfy the discrete-time algebraic Riccati equation

$$
P=A^{T} P A-A^{T} P B\left(R+B^{T} P B\right)^{-1} B^{T} P A+Q .
$$

The above result has a nice intuitive interpretation, namely, the final state weighting matrix $P$ is such that, in the unconstrained case, the horizon 2 cost function is equivalent to the infinite horizon cost [3], [22].

\section{B. First order plants, horizon $N=2$ and $R=0$}

Fig. 1 illustrates the necessary and sufficient conditions (30) and (31). For comparison, we have also included the sufficient condition of [14, Corollary 8], namely:

$$
\frac{Q}{P} \geq A^{2} \text {. }
$$

It can be seen from Fig. 1 that there exists a significant gap between the merely sufficient condition of [14] and the necessary and sufficient condition obtained in the present work.

\section{Near Optimality}

To illustrate the use of Theorem 3 , we choose $N=2, n=$ 1, $A=A^{\prime}=3 / 2, B=B^{\prime}=1, P=P^{\prime}=4, R=R^{\prime}=0$, $Q=3$ and $Q^{\prime}=3-\varepsilon$ for some $0<\varepsilon<3$. Then, according to (30), the horizon one law $\kappa_{1}$ is initially optimal (for the problem with weighting matrix $Q$ ). However, $\kappa_{1}=\kappa_{1}^{\prime}$ is not initially optimal (for the problem with $Q^{\prime}$ ).

In Theorem 3 the relevant matrices are

$$
\begin{gathered}
H_{1}=H_{1}^{\prime}=4, H_{2}=\left(\begin{array}{cc}
12 & 6 \\
6 & 4
\end{array}\right), H_{2}^{\prime}=\left(\begin{array}{cc}
12-\varepsilon & 6 \\
6 & 4
\end{array}\right) \\
F_{1}=F_{1}^{\prime}=3, F_{2}=\left(\begin{array}{c}
18 \\
9
\end{array}\right), F_{2}^{\prime}=\left(\begin{array}{c}
18-3 \varepsilon / 2 \\
9
\end{array}\right) \\
L_{1}=L_{1}^{\prime}=9, L_{2}=26, L_{2}^{\prime}=26-9 \varepsilon / 4 \\
W_{1}=W_{1}^{\prime}=9 / 4, T_{1}=T_{1}^{\prime}=3 / 2 .
\end{gathered}
$$

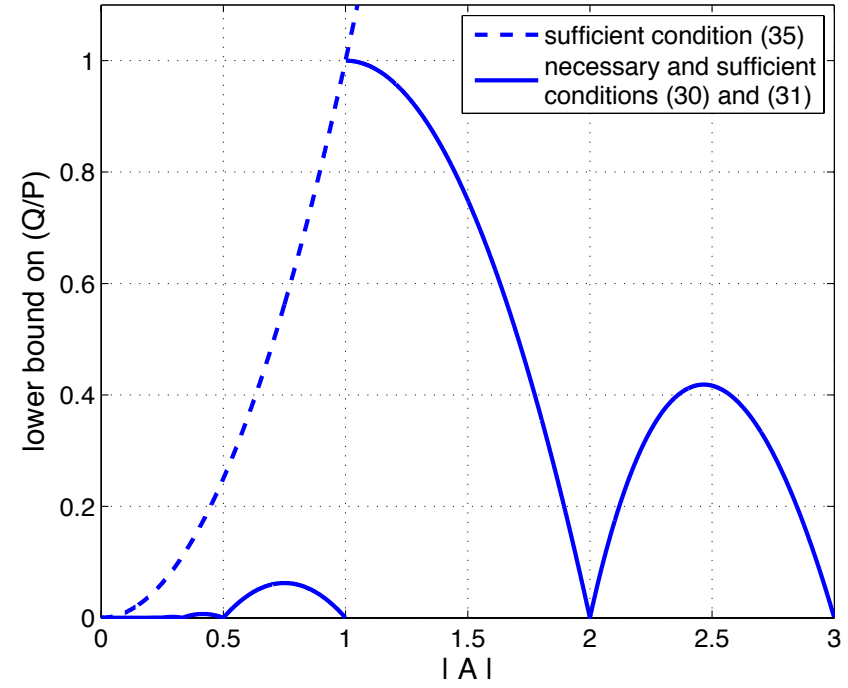

Fig. 1. Necessary and sufficient conditions for the horizon one law to be initially optimal as a function of $|A|$ (solid line). The dashed line shows the sufficient condition of [14].

The smallest eigenvalue of $H_{2}^{\prime}$ is

$$
\varphi_{\varepsilon}=\frac{1}{2}\left(16-\varepsilon-\sqrt{\varepsilon^{2}-16 \varepsilon+208}\right)
$$

and, thus, the relevant constants in Theorem 3 are $\varepsilon_{Q}=\varepsilon$, $\alpha=0$ (since $c=c^{\prime}$ ), so that

$$
\mu=\left|x_{0}\right|, \quad d_{1}=\left|3 x_{0} / 2+u_{0}^{\prime}\right| \leq \delta / 2 .
$$

We also have $\varepsilon_{L_{2}}=9 \varepsilon / 4, \varepsilon_{F_{2}}=3 \varepsilon / 2$, and

$$
\left|T_{2}^{\prime} x_{0}\right| \cdot\left\|H_{2}^{\prime-1 / 2}\right\|_{2} \leq \frac{\left\|F_{2}^{\prime}\right\|_{2}\left|x_{0}\right|}{\varphi_{\varepsilon}},
$$

where $\left\|F_{2}^{\prime}\right\|_{2}^{2}=9 \varepsilon^{2} / 4-54 \varepsilon+405$.

Given the above, Theorem 3 states that the error in applying the control law $\kappa_{1}$ to the problem with weighting $Q^{\prime}=Q-\epsilon$ is less or equal to

$$
\frac{\varepsilon \delta^{2}}{4}+\varepsilon\left|x_{0}\right|^{2}\left(\frac{17}{4}+6 \frac{\left\|F_{2}^{\prime}\right\|_{2}}{\varphi_{\varepsilon}}+4\left(\frac{\left\|F_{2}^{\prime}\right\|_{2}}{\varphi_{\varepsilon}}\right)^{2}\right) \text {. }
$$

\section{CONCLUSIONS}

This paper has established both necessary, and necessary and sufficient conditions for the computationally efficient horizon one quantized control law to provide the solution of model predictive control with a quadratic cost function and a countably infinite input constraint set. The analysis given uses geometric properties of Voronoi partitions induced by lattice vector quantizers and is applicable to LTI systems of arbitrary order. The results show when the simpler horizon one law gives the same input as does a more complex horizon $N$ law. Also, we have shown that "near optimality" holds when the actual system parameters are close to their nominal values. The results have importance in many areas of application, including power electronics [6] and telecommunications, where horizon one laws are inevitably used to meet computational time limits. 
An interesting extension of the situation examined would be to include set-up costs of the type found, for example, in manufacturing applications. This would lead to sparse control problems, which lie outside the current framework, but would be interesting to explore in future work; see also [23]-[26].

\section{APPENDIX}

\section{A. Proof of Lemma 2}

We first note that, if $m \in \mathbb{Z}, w \in \mathbb{R}^{N}$ and $1 \leq k \leq N$, then:

$$
q_{\widetilde{\mathbb{U}}^{N}}\left(w-m \delta H_{N}^{1 / 2} e_{k, N}\right)=q_{\widetilde{U}^{N}}(w)-m \delta H_{N}^{1 / 2} e_{k, N} .
$$

In fact, $z$ is in the left hand side of (36), iff $z \in H_{N}^{1 / 2} \mathbb{U}^{N}$ and $\left|z-\left(w-m \delta H_{N}^{1 / 2} e_{k, N}\right)\right| \leq\left|q-\left(w-m \delta H_{N}^{1 / 2} e_{k, N}\right)\right|$ for all $q \in \mathbb{U}^{N}$, iff $z \in H_{N}^{1 / 2} \mathbb{U}^{N}$ and $\left|\left(z+m \delta H_{N}^{1 / 2} e_{k, N}\right)-w\right| \leq$ $\left|q^{\prime}-w\right|$ for all $q^{\prime} \in \mathbb{U}^{N}$, iff $z$ is in the right hand side of (36).

1) Using Equation (36) we obtain:

$$
\begin{gathered}
w \in M_{k, N}^{*} \Leftrightarrow k \delta \in e_{1, N}^{T} H_{N}^{-1 / 2} q_{\widetilde{\mathbb{U}}^{N}}(w) \\
=e_{1, N}^{T} H_{N}^{-1 / 2} q_{\widetilde{\mathbb{U}}^{N}}\left(w-k \delta H_{N}^{1 / 2} e_{1, N}\right)+k \delta \\
\Leftrightarrow 0 \in e_{1, N}^{T} H_{N}^{-1 / 2} q_{\widetilde{\mathbb{U}}^{N}}\left(w-k \delta H_{N}^{1 / 2} e_{1, N}\right) \\
\Leftrightarrow w-k \delta H_{N}^{1 / 2} e_{1, N} \in M_{0, N}^{*} .
\end{gathered}
$$

2) By 1) it suffices to show that $M_{0, N}^{*}$ is closed. Let $\left(w_{N}\right)_{N} \subset M_{0, N}^{*}$ with $\lim _{n} w_{n}=w$. There exist $q_{2}^{(n)}, \ldots, q_{N}^{(n)} \in \mathbb{U}$ such that, for all $n$ we have

$\left|w_{n}-H_{N}^{1 / 2}\left(0, q_{2}^{(n)}, \ldots, q_{N}^{(n)}\right)^{T}\right| \leq\left|w_{n}-H_{N}^{1 / 2} v\right|, \forall v \in \mathbb{U}^{N}$.

Thus, $\sup _{n}\left|H_{N}^{1 / 2}\left(0, q_{2}^{(n)}, \ldots, q_{N}^{(n)}\right)^{T}\right|<\infty$ and there exists some convergent subsequence, which gives $\lim _{n}\left(q_{2}^{\left(l_{n}\right)}, \ldots, q_{N}^{\left(l_{n}\right)}\right)=\left(q_{2}, \ldots, q_{N}\right)$. This shows $\left|w-H_{N}^{1 / 2}\left(0, q_{2}, \ldots, q_{N}\right)^{T}\right| \leq\left|w-H_{N}^{1 / 2} v\right|$ for all $v \in \mathbb{U}^{N}$. It follows that $w \in M_{0, N}^{*}$.

3) By Equation (36) we have

$w \in M_{0, N}^{*} \Leftrightarrow \exists q_{2}, \ldots, q_{N} \in \mathbb{U}:$

$$
\begin{aligned}
& \left(0, q_{2}, \ldots, q_{N}\right)^{T} \in H_{N}^{-1 / 2} q_{\widetilde{U}^{N}}(w) \\
\Leftrightarrow & H_{N}^{1 / 2}\left(0, q_{2}, \ldots, q_{N}\right)^{T} \\
& \in q_{\widetilde{\mathbb{U}}^{N}}\left(w-\delta \sum_{k=2}^{N} q_{k} H_{N}^{1 / 2} e_{k, N}\right)+\delta \sum_{k=2}^{N} q_{k} H_{N}^{1 / 2} e_{k, N} \\
\Leftrightarrow & 0 \in q_{\widetilde{\mathbb{U}}^{N}}\left(w-\delta \sum_{k=2}^{N} q_{k} H_{N}^{1 / 2} e_{k, N}\right) \\
\Leftrightarrow & w-\delta \sum_{k=2}^{N} q_{k} H_{N}^{1 / 2} e_{k, N} \in C_{0, N}^{*} .
\end{aligned}
$$

4) By 1) and 2) it suffices to show that $M_{0, N}^{*} \subset \overline{\left(M_{0, N}^{*}\right)^{0}}$. Let $z \in M_{0, N}^{*}$. By 3), there exist $q \in C_{0, N}^{*}$ and $m_{2}, \ldots, m_{N} \in$ $\mathbb{Z}$ such that

$$
z=q+\sum_{k=2}^{N} \delta m_{k} H_{N}^{1 / 2} e_{k, N} .
$$

Since $C_{0, N}^{*}$ is convex and $0 \in\left(C_{0, N}^{*}\right)^{0}$, we have

$$
U_{\varepsilon}(q) \cap\left(C_{0, N}^{*}\right)^{0} \neq \emptyset, \quad \forall \varepsilon>0 .
$$

Say, $p_{\varepsilon} \in U_{\varepsilon}(q) \cap\left(C_{0, N}^{*}\right)^{0}$, then

$$
p_{\varepsilon}+\sum_{k=2}^{N} \delta m_{k} H_{N}^{1 / 2} e_{k, N} \in U_{\varepsilon}(z) \cap\left(M_{0, N}^{*}\right)^{0},
$$

yielding

$$
z=\lim _{n}\left(p_{1 / n}+\sum_{k=2}^{N} \delta m_{k} H_{N}^{1 / 2} e_{k, N}\right) \in \overline{\left(M_{0, N}^{*}\right)^{0}} .
$$

5) Let $z \in\left(M_{k, N}^{*}\right)^{o} \cap M_{l, N}^{*}$. Then, there exist $q_{2}, \ldots, q_{N} \in$ $\mathbb{U}$ such that

$$
\left|z-H_{N}^{1 / 2}\left(l \delta, q_{2}, \ldots, q_{N}\right)^{T}\right| \leq\left|z-H_{N}^{1 / 2} w\right|, \quad \forall w \in \mathbb{U}^{N},
$$

and there exists $\mu>0$ such that $\forall \varepsilon \in(-\mu, \mu)$ there exist $q_{2}^{(\varepsilon)}, \ldots, q_{N}^{(\varepsilon)} \in \mathbb{U}$ which give:

$$
\begin{aligned}
& \left|z-\varepsilon H_{N}^{-1 / 2} e_{1, N}-H_{N}^{1 / 2}\left(k \delta, q_{2}^{(\varepsilon)}, \ldots, q_{N}^{(\varepsilon)}\right)^{T}\right| \\
& \quad \leq\left|z-\varepsilon H_{N}^{-1 / 2} e_{1, N}-H_{N}^{1 / 2} w\right|, \quad \forall w \in \mathbb{U}^{N} .
\end{aligned}
$$

Use of (37) provides

$$
\begin{aligned}
& \mid z-\left.H_{N}^{1 / 2}\left(l \delta, q_{2}, \ldots, q_{N}\right)^{T}\right|^{2} \\
& \leq \mid z-\left.H_{N}^{1 / 2}\left(k \delta, q_{2}^{(\varepsilon)}, \ldots, q_{N}^{(\varepsilon)}\right)^{T}\right|^{2} \\
&=\mid z-\varepsilon H_{N}^{-1 / 2} e_{1, N}-H_{N}^{1 / 2}\left(k \delta, q_{2}^{(\varepsilon)}, \ldots, q_{N}^{(\varepsilon)}\right)^{T}\left.\quad \varepsilon H_{N}^{-1 / 2} e_{1, N}\right|^{2} \\
&=\left|z-\varepsilon H_{N}^{-1 / 2} e_{1, N}-H_{N}^{1 / 2}\left(k \delta, q_{2}^{(\varepsilon)}, \ldots, q_{N}^{(\varepsilon)}\right)^{T}\right|^{2} \\
& \quad+2 \varepsilon e_{1, N}^{T} H_{N}^{-1 / 2}\left(z-\varepsilon H_{N}^{-1 / 2} e_{1, N}\right. \\
&\left.\quad-H_{N}^{1 / 2}\left(k \delta, q_{2}^{(\varepsilon)}, \ldots, q_{N}^{(\varepsilon)}\right)^{T}\right)+\varepsilon^{2}\left|H_{N}^{-1 / 2} e_{1, N}\right|^{2} \\
& \leq\left|z-H_{N}^{1 / 2}\left(l \delta, q_{2}, \ldots, q_{N}\right)^{T}\right|^{2} \\
& \quad-2 \varepsilon e_{1, N}^{T} H_{N}^{-1 / 2}\left(z-H_{N}^{1 / 2}\left(l \delta, q_{2}, \ldots, q_{N}\right)^{T}\right) \\
& \quad+2 \varepsilon^{2}\left|H_{N}^{-1 / 2} e_{1, N}\right|^{2}+2 \varepsilon e_{1, N}^{T} H_{N}^{-1 / 2} \\
& \quad\left(z-\varepsilon H_{N}^{-1 / 2} e_{1, N}-H_{N}^{1 / 2}\left(k \delta, q_{2}^{(\varepsilon)}, \ldots, q_{N}^{(\varepsilon)}\right)^{T}\right) \\
&=\left|z-H_{N}^{1 / 2}\left(l \delta, q_{2}, \ldots, q_{N}\right)^{T}\right|^{2}+2 \varepsilon l \delta-2 \varepsilon k \delta .
\end{aligned}
$$

This shows that $\varepsilon k \leq \varepsilon l$ for all $\varepsilon \in(-\mu, \mu)$. We thus have $k=l$, which proves the result.

\section{B. Proof of Lemma 3}

If $x \in \operatorname{Ker} B^{T} P A$, then we have, $\forall t \in \mathbb{R}$,

$$
\begin{aligned}
0= & \kappa_{1}(t x)=e_{1, N}^{T} H_{N}^{-1 / 2} q_{\widetilde{\mathbb{U}}^{N}}\left(-t H_{N}^{-1 / 2} F_{N} x\right), \forall t \in \mathbb{R} \\
& \Rightarrow-t H_{N}^{-1 / 2} F_{N} x \in M_{0, N}^{*}, \forall t \in \mathbb{R} \\
& \Rightarrow H_{N}^{-1 / 2} F_{N} x \in \operatorname{Span}\left(H_{N}^{1 / 2} e_{2, N}, \ldots, H_{N}^{1 / 2} e_{N, N}\right),
\end{aligned}
$$

by 3) of Lemma 2. If, moreover, $F_{N}$ is onto and $\operatorname{dim} F_{N}\left(\operatorname{Ker} B^{T} P A\right)<N-1$, then $\operatorname{rank} F_{N}<N$, a contradiction. 


\section{Proof of Lemma 4}

Define $A_{\mu}:=\left\{z \in \mathbb{R}^{n} \mid-B^{T} P A z / \gamma=\mu\right\}$. Then $Q_{k}=$ $\bigcup_{\mu \in L_{k}} A_{\mu}$, and

$$
\begin{aligned}
A_{\mu} & =\left\{z \in \mathbb{R}^{n} \mid-\frac{B^{T} P A}{\gamma}\left(z+\frac{\mu \gamma A^{T} P B}{\left|A^{T} P B\right|^{2}}\right)=0\right\} \\
& =-\frac{\mu \gamma A^{T} P B}{\left|A^{T} P B\right|^{2}}+\operatorname{Ker} B^{T} P A,
\end{aligned}
$$

which implies that

$$
\begin{aligned}
- & H_{N}^{-1 / 2} F_{N} Q_{k}=-\bigcup_{\mu \in L_{k}} H_{N}^{-1 / 2} F_{N} A_{\mu} \\
& =\bigcup_{\mu \in L_{k}}\left[H_{N}^{-1 / 2} F_{N} \frac{\mu \gamma A^{T} P B}{\left|A^{T} P B\right|^{2}}+H_{N}^{-1 / 2} F_{N}\left(\operatorname{Ker} B^{T} P A\right)\right] \\
& =H_{N}^{-1 / 2} F_{N}\left(\operatorname{Ker} B^{T} P A\right)+L_{k} w_{N} .
\end{aligned}
$$

If the horizon one law is initially optimal, then we have (by Theorem 1 and Lemma 2)

$$
\begin{aligned}
& H_{N}^{-1 / 2} F_{N} \operatorname{Ker}\left(B^{T} P A\right)+L_{k} w_{N} \\
\subset & C_{0, N}^{*}+k \delta H_{N}^{1 / 2} e_{1, N}+\left\{\sum_{l=2}^{N} \delta m_{l} H_{N}^{1 / 2} e_{l, N} \mid m_{l} \in \mathbb{Z}\right\},
\end{aligned}
$$

so that

$k \delta\left(w_{N}-H_{N}^{1 / 2} e_{1, N}\right) \in C_{0, N}^{*}+\left\{\sum_{l=2}^{N} \delta m_{l} H_{N}^{1 / 2} e_{l, N} \mid m_{l} \in \mathbb{Z}\right\}$.

This amounts to

$\forall k \in \mathbb{Z}, \exists z \in C_{0, N}^{*}$ and $m_{2}, \ldots, m_{N} \in \mathbb{Z}:$

$$
k \delta\left(H_{N}^{-1 / 2} w_{N}-e_{1, N}\right)=H_{N}^{-1 / 2} z+\sum_{l=2}^{N} \delta m_{l} e_{l, N} .
$$

Thus $e_{1, N}^{T} H_{N}^{-1 / 2} w_{N}-1=\frac{1}{k \delta} H_{N}^{-1 / 2} z$ and, since $C_{0, N}^{*}$ is bounded, this converges to 0 as $k \rightarrow \infty$.

\section{Proof of Lemma 5}

First we show that there is some finite $M \subset \mathbb{U}^{N} \backslash\{0\}$ such that $C_{0, N}^{*}=\bigcap_{\vec{k} \in M} J_{\vec{k}}$. Define

$$
S:=\left(\bigcap_{m=1}^{N} J_{\delta e_{m, N}}\right) \cap\left(\bigcap_{m=1}^{N} J_{-\delta e_{m, N}}\right) .
$$

Clearly, $S$ is bounded, since if $x \in S$ then

$$
\left|2 x^{T} H_{N}^{1 / 2} e_{m, N}\right| \leq \delta\left|H_{N}^{1 / 2} e_{m, N}\right|^{2}, \forall 1 \leq m \leq N .
$$

Thus,

$$
\begin{aligned}
\frac{|x|^{2}}{\left\|H_{N}^{-1 / 2}\right\|^{2}} & \leq\left|H_{N}^{1 / 2} x\right|^{2}=\sum_{m=1}^{N}\left|\left(H_{N}^{1 / 2} x\right)_{m}\right|^{2} \\
& =\sum_{m=1}^{N}\left|x^{T} H_{N}^{1 / 2} e_{m, N}\right|^{2} \leq \frac{\delta^{2}}{4} \sum_{m=1}^{N}\left|H_{N}^{1 / 2} e_{m, N}\right|^{4}
\end{aligned}
$$

which shows that $\sup _{x \in S}|x|<\infty$. Consequently, $S$ is contained in the the open ball $U_{R}(0)$ centered at 0 with radius $R$ for some $R>0$. Now, define

$$
\begin{aligned}
& M=\left\{\vec{k} \in \mathbb{U}^{N} \backslash\{0\} \mid \vec{k}=\delta e_{m, N} \text { for some } m\right. \\
& \left.\quad \text { or } \vec{k}=-\delta e_{m, N} \text { for some } m \text { or } S \not \subset J_{\vec{k}}\right\} .
\end{aligned}
$$

Clearly, $M$ is finite, since if

$$
\vec{k} \in M \backslash\left\{ \pm \delta e_{m, N} \mid 1 \leq m \leq N\right\},
$$

then

$S \not \subset J_{\vec{k}} \Rightarrow U_{R}(0) \not \subset J_{\vec{k}} \Rightarrow \operatorname{dist}\left(0, J_{\vec{k}}\right)<R \Rightarrow(\sigma / 2)|\vec{k}|<R$, where $\sigma$ is the smallest eigenvalue of $H_{N}^{1 / 2}$. Note that $\operatorname{dist}\left(0, J_{\vec{k}}\right)=\frac{1}{2}\left|H_{N}^{1 / 2} \vec{k}\right| \geq \frac{\sigma}{2}|\vec{k}|$.

Finally, if $z \in \bigcap_{\vec{k} \in M} J_{\vec{k}}$ and $\vec{l} \in \mathbb{U}^{N} \backslash M$ then $z \in S \subset$ $J_{\vec{l}}$, thus $z \in \bigcap_{\vec{k} \in \mathbb{U}^{N}} J_{\vec{k}}=C_{0, N}^{*}$. This shows that $C_{0, N}^{*}=$ $\bigcap_{\vec{k} \in M} J_{\vec{k}}$.

Let $M=\left\{\overrightarrow{k_{1}}, \ldots, \overrightarrow{k_{r}}\right\}$. There exist $\mu_{k}^{(i)} \in \mathbb{R}$ such that $H_{N}^{1 / 2} \overrightarrow{k_{i}}=\sum_{k=1}^{N} \mu_{k}^{(i)} b_{k}$ for all $1 \leq i \leq r$.

If $\lambda_{k} \in[-c, c]$ and $\alpha_{1}, \alpha_{2} \in \mathbb{R}$ with $\alpha_{1} \neq \alpha_{2}$ and $\overrightarrow{k_{i}} \in M$ such that

$$
\mu b_{1}+\alpha_{l} \sum_{k=2}^{N} \lambda_{k} b_{k} \in \partial J_{\overrightarrow{k_{i}}}, \quad \forall l \in\{1,2\} .
$$

Then, we have

$$
\begin{array}{r}
\left(\mu b_{1}+\left(\xi \alpha_{1}+(1-\xi) \alpha_{2}\right) \sum_{k=2}^{N} \lambda_{k} b_{k}-\frac{1}{2} H_{N}^{1 / 2} \overrightarrow{k_{i}}\right)^{T} H_{N}^{1 / 2} \overrightarrow{k_{i}} \\
=0, \quad \forall \xi \in \mathbb{R} .
\end{array}
$$

Thus, and since $M$ is finite and $\partial C_{0, N}^{*} \subset \bigcup_{\vec{k} \in M} \partial J_{\vec{k}}$, there exists $I:[-c, c]^{N-1} \rightarrow\{1, \ldots, r\}$ :

$$
\begin{aligned}
\left(2 \mu b_{1}+2 \alpha \sum_{k=2}^{N} \lambda_{k} b_{k}-H_{N}^{1 / 2} \vec{k}_{I\left(\lambda_{2}, \ldots, \lambda_{N}\right)}\right)^{T} H_{N}^{1 / 2} \vec{k}_{I\left(\lambda_{2}, \ldots, \lambda_{N}\right)} & \\
=0 & \text { for all } \alpha \in \mathbb{R}
\end{aligned}
$$

and, consequently, $\sum_{k=2}^{N} \lambda_{k} \mu_{k}^{\left(I\left(\lambda_{2}, \ldots, \lambda_{N}\right)\right)}=0$. Let $\operatorname{Im} I=$ $\left\{i_{1}, \ldots, i_{l}\right\}$ for some $1 \leq i_{1}<\ldots<i_{l} \leq r$, define $a_{j}^{T}=$ $\left(\mu_{2}^{\left(i_{j}\right)}, \ldots, \mu_{N}^{\left(i_{j}\right)}\right)$ if $1 \leq j \leq l$, and $\mathcal{A}=\left(a_{1} \ldots a_{l}\right)^{T} \in$ $\mathbb{R}^{l \times N-1}$. We have shown that if $\left(\lambda_{2}, \ldots, \lambda_{N}\right) \in[-c, c]^{N-1}$, then at least one entry of the vector $\mathcal{A}\left(\begin{array}{lll}\lambda_{2} & \ldots & \lambda_{N}\end{array}\right)^{T}$ equals 0 . If we define $v_{t}=\left(t, t^{2} \ldots, t^{N}\right)^{T}$ we therefore see that the polynomial $\Pi_{k=1}^{l}\left(a_{k}^{T} v_{t}\right)$ vanishes on some neighborhood of $0 \in \mathbb{R}$. Consequently, there exists some $s \in\{1, \ldots, l\}$ such that the polynomial $a_{s}^{T} v_{t}$ and, thus, $a_{s}$ vanishes. This shows that $H_{N}^{1 / 2} \vec{k}_{i_{s}}=\mu_{1}^{\left(i_{s}\right)} b_{1}$.

Finally, we note that there exists some $\left(\lambda_{2}, \ldots, \lambda_{N}\right) \in$ $[-c, c]^{N-1}$ such that $I\left(\lambda_{2}, \ldots, \lambda_{N}\right)=i_{s}$. Hence,

$$
\left(2 \mu b_{1}-H_{N}^{1 / 2} \vec{k}_{i_{s}}\right)^{T} H_{N}^{1 / 2} \vec{k}_{i_{s}}=0,
$$

so that $0=\mu_{1}^{\left(i_{s}\right)}\left|b_{1}\right|^{2}\left(2 \mu-\mu_{1}^{\left(i_{s}\right)}\right)$. Since both $\left|b_{1}\right|$ and $\mu_{1}^{\left(i_{s}\right)}$ do not vanish, we obtain $2 \mu b_{1}=H_{N}^{1 / 2} \vec{k}_{i_{s}}$. 


\section{E. Proof of Theorem 2}

Necessity: Initial optimality $\Rightarrow 2$ ) is Lemma 3 , and initial optimality $\Rightarrow 3$ ) follows from Lemma 4 . We next show that initial optimality $\Rightarrow 1$ ).

Since, by Lemma 7, $S_{N} \subset M_{0, N}^{*}$ we see, by Lemmas 2 and 6 , that $\forall \lambda_{1} \in L_{0}, \forall \lambda_{2}, \ldots, \lambda_{N} \in \mathbb{R}$ there exist $m_{2}, \ldots, m_{N} \in$ $\mathbb{Z}$ such that

$$
\sum_{k=1}^{N} \lambda_{k} q_{N}^{(k)}+\sum_{j=2}^{N} \delta m_{j} H_{N}^{1 / 2} e_{j, N} \in C_{0, N}^{*} .
$$

This gives

$$
\begin{aligned}
& \left|\sum_{k=1}^{N} \lambda_{k} q_{N}^{(k)}+\delta H_{N}^{1 / 2}\left(0, m_{2}, \ldots, m_{N}\right)^{T}\right| \\
& \quad \leq\left|\sum_{k=1}^{N} \lambda_{k} q_{N}^{(k)}+\delta H_{N}^{1 / 2}\left(0, m_{2}, \ldots, m_{N}\right)^{T}-H_{N}^{1 / 2} v\right|,
\end{aligned}
$$

for all $v \in \mathbb{U}^{N}$. If we choose $v=\left(0, \delta m_{2}, \ldots, \delta m_{N}\right)^{T}$, then we obtain

$$
\begin{aligned}
2 \delta\left(\sum_{k=1}^{N} \lambda_{k} q_{N}^{(k)}\right)^{T} & H_{N}^{1 / 2}\left(0, m_{2}, \ldots, m_{N}\right)^{T} \\
& +\delta^{2}\left|H_{N}^{1 / 2}\left(0, m_{2}, \ldots, m_{N}\right)^{T}\right|^{2} \leq 0 .
\end{aligned}
$$

By equation (21) we have $q_{N}^{(1)^{T}} H_{N}^{1 / 2} e_{j, N}=0$ if $j>1$. Thus, the Cauchy-Schwarz-inequality provides

$$
\begin{aligned}
0 \geq & \left(2 \sum_{k=2}^{N} \lambda_{k} q_{N}^{(k)}\right)^{T} H_{N}^{1 / 2}\left(0, m_{2}, \ldots, m_{N}\right)^{T} \\
& +\delta\left|H_{N}^{1 / 2}\left(0, m_{2}, \ldots, m_{N}\right)^{T}\right|^{2} \\
\geq \delta & \left.H_{N}^{1 / 2}\left(0, m_{2}, \ldots, m_{N}\right)^{T}\right|^{2} \\
& -2\left|\sum_{k=2}^{N} \lambda_{k} q_{N}^{(k)}\right| \cdot\left|H_{N}^{1 / 2}\left(0, m_{2}, \ldots, m_{N}\right)^{T}\right| .
\end{aligned}
$$

Now, define

$$
\begin{aligned}
\Gamma & :=\min _{\left(m_{2}, \ldots, m_{N}\right) \in \mathbb{Z}^{N-1} \backslash\{0\}} \delta\left|H_{N}^{1 / 2}\left(0, m_{2}, \ldots, m_{N}\right)^{T}\right| \\
& \geq \frac{\delta}{\left\|H_{N}^{-1 / 2}\right\|} \min _{\left(m_{2}, \ldots, m_{N}\right) \in \mathbb{Z}^{N-1} \backslash\{0\}}\left|\left(0, m_{2}, \ldots, m_{N}\right)^{T}\right|>0,
\end{aligned}
$$

and choose $\widetilde{c}>0$ such that

$$
2\left|\sum_{k=2}^{N} \lambda_{k} q_{N}^{(k)}\right|<\Gamma, \quad \forall\left(\lambda_{2}, \ldots, \lambda_{k}\right) \in[-\widetilde{c}, \widetilde{c}]^{N-1} .
$$

If $\left(\lambda_{2}, \ldots, \lambda_{k}\right) \in[-\widetilde{c}, \widetilde{c}]^{N-1}$ and $\lambda_{1} \in L_{0}$ we have $m_{2}=$ $\ldots=m_{N}=0$, since, otherwise we would have

$$
0 \geq \delta\left|H_{N}^{1 / 2}\left(0, m_{2}, \ldots, m_{N}\right)^{T}\right|-2\left|\sum_{k=2}^{N} \lambda_{k} q_{N}^{(k)}\right|,
$$

which would imply that

$$
\Gamma>2\left|\sum_{k=2}^{N} \lambda_{k} q_{N}^{(k)}\right| \geq \delta\left|H_{N}^{1 / 2}\left(0, m_{2}, \ldots, m_{N}\right)^{T}\right| \geq \Gamma,
$$

a contradiction. Therefore, it holds that $\sum_{k=1}^{N} \lambda_{k} q_{N}^{(k)} \in C_{0, N}^{*}$, and if $\left(\mu_{2}, \ldots, \mu_{N}\right) \in[-c, c]^{N-1}$, where

$$
c:=\widetilde{c} \cdot \min _{2 \leq k \leq N}\left|q_{N}^{(k)}\right|,
$$

then

$$
\frac{\delta}{2\left|H_{N}^{-1 / 2} e_{1, N}\right|}\left(\left|H_{N}^{-1 / 2} e_{1, N}\right| q_{N}^{(1)}\right)+\sum_{k=2}^{N} \mu_{k} \frac{q_{N}^{(k)}}{\left|q_{N}^{(k)}\right|} \in C_{0, N}^{*} .
$$

Since the left hand side of (39) lies on $\partial S_{N}=\partial M_{0, N}^{*}$ it is not an inner point of $C_{0, N}^{*}$. By Lemma 9, there exists some $p \in \mathbb{U}^{N}$ such that $\delta q_{N}^{(1)}=H_{N}^{1 / 2} p$, which gives 1$)$.

Sufficiency: By Lemma 2, we have

$$
\begin{aligned}
M_{k, N}^{*}= & \bigcap_{\vec{k} \in \mathbb{U}^{N} \backslash\{0\}} J_{\vec{k}} \\
& +\left\{k \delta H_{N}^{1 / 2} e_{1, N}+\sum_{k=2}^{N} \delta l_{k} H_{N}^{1 / 2} e_{k, N} \mid l_{k} \in \mathbb{Z}\right\} .
\end{aligned}
$$

Since

$$
w:=\frac{H_{N}^{-1} e_{1, N}}{e_{1, N}^{T} H_{N}^{-1} e_{1, N}} \in\{1\} \times \mathbb{Z}^{N-1},
$$

we have $\pm \delta w \in \mathbb{U}^{N} \backslash\{0\}$ and, therefore,

$$
\begin{aligned}
M_{k, N}^{*} \subset & \left(J_{\delta w} \cap J_{-\delta w}\right) \\
& +\left\{k \delta H_{N}^{1 / 2} e_{1, N}+\sum_{k=2}^{N} \delta l_{k} H_{N}^{1 / 2} e_{k, N} \mid l_{k} \in \mathbb{Z}\right\} .
\end{aligned}
$$

We next show that the latter set is contained in $H_{N}^{1 / 2} \operatorname{Span}\left(e_{2, N}, \ldots, e_{N, N}\right)+\overline{L_{k}} H_{N}^{1 / 2} w$, i.e., if $q \in J_{\delta w} \cap$ $J_{-\delta w}$, then

$$
q+k \delta H_{N}^{1 / 2} e_{1, N} \in H_{N}^{1 / 2} \operatorname{Span}\left(e_{2, N}, \ldots, e_{N, N}\right)+\overline{L_{k}} H_{N}^{1 / 2} w .
$$

For that purpose we note that if $H_{N}^{1 / 2} w$ is perpendicular to $H_{N}^{1 / 2} \operatorname{Span}\left(e_{2, N}, \ldots, e_{N, N}\right)$, then there exist $\mu, \lambda_{2}, \ldots, \lambda_{N} \in$ $\mathbb{R}$ such that

$$
q+k \delta H_{N}^{1 / 2} e_{1, N}=\mu H_{N}^{1 / 2} w+\sum_{m=2}^{N} \lambda_{m} H_{N}^{1 / 2} e_{m, N} .
$$

On the other hand, since $q \in J_{\delta w} \cap J_{-\delta w}$, we have $\left|2 q^{T} H_{N}^{1 / 2} w\right| \leq \delta w^{T} H_{N} w$ and therefore:

$$
\begin{aligned}
\delta w^{T} H_{N} w \geq & \mid 2\left(-k \delta e_{1, N}^{T} H_{N}^{1 / 2}+\mu w^{T} H_{N}^{1 / 2}\right. \\
& \left.+\sum_{m=2}^{N} \lambda_{m} e_{m, N}^{T} H_{N}^{1 / 2}\right) H_{N}^{1 / 2} w \mid \\
= & -2 k \delta e_{1, N}^{T} H_{N} w+2 \mu w^{T} H_{N} w \mid .
\end{aligned}
$$

Since $w^{T} H_{N} w=\left(e_{1, N}^{T} H_{N}^{-1} e_{1, N}\right)^{-1}=e_{1, N}^{T} H_{N} w$, we obtain that $\mu \in \overline{L_{k}}$.

Define

$$
Y_{k}:=H_{N}^{1 / 2} \operatorname{Span}\left(e_{2, N}, \ldots, e_{N, N}\right)+L_{k}^{0} H_{N}^{1 / 2} w .
$$

We have shown that $\left(M_{k, N}^{*}\right)^{0} \subset Y_{k}$ for all $k \in \mathbb{Z}$. Now it follows that $Y_{k} \subset M_{k, N}^{*}$. In fact, if $z \in Y_{k} \backslash M_{k, N}^{*}$ then there 
exists some $l \in \mathbb{Z} \backslash\{k\}$ such that $z \in M_{l, N}^{*}$, so that $z \in \overline{Y_{l}}$. There are $\alpha_{k}, \beta_{k} \in \mathbb{R}, \mu \in \overline{L_{l}}$ and $\lambda \in L_{k}^{0}$ :

$z=\sum_{k=2}^{N} \alpha_{k} H_{N}^{1 / 2} e_{k, N}+\mu H_{N}^{1 / 2} w=\sum_{k=2}^{N} \beta_{k} H_{N}^{1 / 2} e_{k, N}+\lambda H_{N}^{1 / 2} w$.

Thus, $\mu=\lambda$ and $l=k$, a contradiction. Therefore, $M_{k, N}^{*}=$ $\overline{Y_{k}}$ for all $k \in \mathbb{Z}$ by Lemma 2 .

Finally, we will show that $-H_{N}^{-1 / 2} F_{N} Q_{k} \subset \overline{Y_{k}}$. By Lemma 4 and by Condition 2) we have

$$
-H_{N}^{-1 / 2} F_{N} Q_{k}=H_{N}^{1 / 2} \operatorname{Span}\left(e_{2, N}, \ldots, e_{N, N}\right)+L_{k} w_{N} .
$$

Thus, we only need to show that

$$
w_{N} \in H_{N}^{1 / 2} w+H_{N}^{1 / 2} \operatorname{Span}\left(e_{2, N}, \ldots, e_{N, N}\right) .
$$

Since $\left[H_{N}^{1 / 2} \operatorname{Span}\left(e_{2, N}, \ldots, e_{N, N}\right)\right]^{\perp}=\operatorname{Span} H_{N}^{-1 / 2} e_{1, N}$, this amounts to showing that the Euclidean projection of $w_{N}$ on $\operatorname{Span} H_{N}^{-1 / 2} e_{1, N}$ equals $H_{N}^{1 / 2} w$. This is, indeed, true, since ${ }^{1} e_{1, N}^{T} H_{N}^{-1 / 2} w_{N} H_{N}^{-1 / 2} e_{1, N} /\left|H_{N}^{-1 / 2} e_{1, N}\right|^{2}=$ $H_{N}^{-1 / 2} e_{1, N} /\left(e_{1, N}^{T} H_{N}^{-1} e_{1, N}\right)$. The result now follows from Theorem 1.

\section{F. Proof of Lemma 8}

We only have to show the 'if'-direction. For that purpose, we show that

a) $Q_{k}^{0}+\frac{\delta k \gamma}{B^{T} P B} \mu=Q_{0}^{0} \quad$ for all $k \in \mathbb{Z}$.

b) $H_{N}^{-1} F_{N} \mu=e_{1, N}-R H_{N}^{-1} e_{1, N}$.

To prove a), we let $w \in Q_{k}^{0}$, i.e.,

$$
-\left(B^{T} P A w\right) / \gamma \in((k-1 / 2) \delta,(k+1 / 2) \delta) .
$$

We then have

$$
\begin{aligned}
-\left(B^{T} P A\right) / \gamma & \left(w+\delta k \gamma \mu /\left(B^{T} P B\right)\right) \\
& =-\left(B^{T} P A w\right) / \gamma-\delta k \in(-\delta / 2, \delta / 2) .
\end{aligned}
$$

Consequently, it holds that $w+\delta k \gamma \mu /\left(B^{T} P B\right) \in Q_{0}^{0}$. The rest is easy to prove.

To prove $\mathrm{b}$ ), we first note that

$$
\begin{aligned}
& F_{N} \mu=\Phi_{N}^{T} \widehat{Q}_{N} \Lambda_{N} \mu \\
& \Lambda_{N} \mu=\left(\begin{array}{c}
A \mu \\
\vdots \\
A^{N} \mu
\end{array}\right)=\left(\begin{array}{c}
B \\
A B \\
\vdots \\
A^{N-1} B
\end{array}\right)=\Phi_{N} e_{1, N} .
\end{aligned}
$$

This gives

$$
\begin{aligned}
F_{N} \mu & =\Phi_{N}^{T} \widehat{Q}_{N} \Phi_{N} e_{1, N}=\left(H_{N}-\widehat{R}_{N}\right) e_{1, N} \\
& =H_{N} e_{1, N}-R e_{1, N} .
\end{aligned}
$$

We, thus, have

$$
\begin{gathered}
-H_{N}^{-1 / 2} F_{N} Q_{k}^{0}=-H_{N}^{-1 / 2} F_{N} Q_{0}^{0}+H_{N}^{-1 / 2} F_{N}\left(\frac{\delta k \gamma}{B^{T} P B} \mu\right) \\
=-H_{N}^{-1 / 2} F_{N} Q_{0}^{0}+\delta k H_{N}^{1 / 2} e_{1, N}(\text { since } R=0) \\
\subset M_{0, N}^{*}+\delta k H_{N}^{1 / 2} e_{1, N}=M_{k, N}^{*},
\end{gathered}
$$

where we have used Lemma 2. The assertion now follows from Theorem 1.

\footnotetext{
${ }^{1}$ Recall that $e_{1, N}^{T} H_{N}^{-1 / 2} w_{N}=1$, by Condition 3$)$.
}

\section{G. Proof of Corollary 2}

Define $y_{k}:=-k R\left(E_{N}-\gamma H_{N}^{-1}\right) e_{1, N} / B^{T} P B$ and let $w=0$ in (37). Let $m_{k} \in \mathbb{Z}^{N-1}$ be any minimizer of $G_{y_{k}}$. Thus, if $k \in \mathbb{Z}$, then the ellipsoid with center $y_{k}+\left(\begin{array}{c}0 \\ m_{k}\end{array}\right)$ and half axis $\sqrt{r_{y_{k}} / d_{i}} v_{i}, 1 \leq i \leq N$, does not contain a point of $\mathbb{Z}^{N}$ in its interior, see Lemma 9. Consequently, we have $\sup _{k} r_{y_{k}}<\infty$, so that $\sup _{k}\left|e_{1, N}^{T} y_{k}\right|<\infty$, from where (27) follows. Next, we show that

$$
H_{N-1}=\left(0 \mid E_{N-1}\right) H_{N}\left(\begin{array}{c}
0^{T} \\
E_{N-1}
\end{array}\right)
$$

where $0 \in \mathbb{R}^{N}$. To this end we recall that if $C$ is some $n \times m$ matrix and the matrices 0 have appropriate dimensions, then

$\left(0 \mid E_{n-k}\right) C \in \mathbb{R}^{n-k \times m}$ removes the upper $k$ rows of $C$,

$C\left(\begin{array}{c}0 \\ E_{m-k}\end{array}\right) \in \mathbb{R}^{n \times m-k}$ removes the left $k$ columns of $C$,

$$
\left(E_{n-k} \mid 0\right) C \in \mathbb{R}^{n-k \times m} \text { removes the lower } k \text { rows of } C,
$$

$C\left(\begin{array}{c}E_{m-k} \\ 0\end{array}\right) \in \mathbb{R}^{n \times m-k}$ removes the right $k$ columns of $C$.

The above gives

$$
\left(E_{n} \mid 0\right) \Phi_{N}\left(\begin{array}{c}
0^{T} \\
E_{N-1}
\end{array}\right)=(B, 0, \ldots, 0)\left(\begin{array}{c}
0^{T} \\
E_{N-1}
\end{array}\right)=0,
$$

so that

$$
\begin{aligned}
H_{N-1}- & \widehat{R}_{N-1}=\Phi_{N-1}^{T} \widehat{Q}_{N-1} \Phi_{N-1} \\
= & \left(0 \mid E_{N-1}\right) \Phi_{N}^{T}\left[\left(\begin{array}{c}
0 \\
E_{(N-1) n}
\end{array}\right) \widehat{Q}_{N-1}\left(0 \mid E_{(N-1) n}\right)\right] \\
& \Phi_{N}\left(\begin{array}{c}
0^{T} \\
E_{N-1}
\end{array}\right) \\
= & \left(0 \mid E_{N-1}\right) \Phi_{N}^{T} \cdot \widetilde{Q} \cdot \Phi_{N}\left(\begin{array}{c}
0^{T} \\
E_{N-1}
\end{array}\right),
\end{aligned}
$$

where $\widetilde{Q}=\widehat{Q}_{N}-\left(\begin{array}{c}E_{n} \\ 0\end{array}\right) Q\left(E_{n} \mid 0\right)$ is $\widehat{Q}_{N-1}$ after adding $n$ rows of zeros to the top, and $n$ columns of zeros to the left. Using (38), we obtain

$$
\begin{aligned}
H_{N-1}-\left(0 \mid E_{N-1}\right) \widehat{R}_{N}\left(\begin{array}{c}
0^{T} \\
E_{N-1}
\end{array}\right) \\
=\left(0 \mid E_{N-1}\right) \Phi_{N}^{T} \widehat{Q}_{N} \Phi_{N}\left(\begin{array}{c}
0^{T} \\
E_{N-1}
\end{array}\right),
\end{aligned}
$$

which gives (40). On the other hand, Cramer's rule shows that

$$
e_{1, N}^{T} H_{N}^{-1} e_{1, N}=\operatorname{det} H_{N-1} / \operatorname{det} H_{N},
$$

and now $R\left(\operatorname{det} H_{N}-\gamma \operatorname{det} H_{N-1}\right)=0$ follows from (11).

Finally, we have

$\operatorname{det} H_{N}-\gamma \operatorname{det} H_{N-1}=\left[\left(H_{N}\right)_{1,1}-\gamma\right] \operatorname{det} H_{N-1}+\operatorname{Pol}(R)$, where $\mathrm{Pol}$ is some polynomial of degree at most $N-2$ and $\left(H_{N}\right)_{1,1}$ is the top left entry of $H_{N}$. Since

$$
\left(H_{N}\right)_{1,1}-\gamma=\left(\Phi_{N}^{T} \widehat{Q}_{N} \Phi_{N}\right)_{1,1}-B^{T} P B
$$

is independent of $R$, the assertion follows. 


\section{H. Proof of Theorem 3}

Suppose $u_{0}^{\prime}=k \delta$ for some $k \in \mathbb{Z}$. Define $c^{T}=-B^{T} P A / \gamma$ and let $\hat{x}_{0}$ (which in general differs from $x_{0}$ ) be the solution of the problem stated in Lemma 11. Then there is some optimal control $u^{*}$ of $V_{N}\left(\hat{x}_{0}, \cdot\right)$ in $\mathbb{U}^{N}$ with $u_{0}^{*}=u_{0}^{\prime}$. Hence, we can bound

$$
\begin{aligned}
& V_{N}^{\prime}\left(x_{0}, u^{\prime}\right)-V_{N}^{\prime}\left(x_{0}, \bar{u}^{\prime}\right)=V_{N}^{\prime}\left(x_{0}, u^{\prime}\right)-V_{N}\left(\hat{x}_{0}, u^{*}\right) \\
& +V_{N}\left(\hat{x}_{0}, u^{*}\right)-V_{N}\left(\hat{x}_{0}, \bar{u}^{\prime}\right)+V_{N}\left(\hat{x}_{0}, \bar{u}^{\prime}\right)-V_{N}^{\prime}\left(x_{0}, \bar{u}^{\prime}\right) \\
& \leq \underbrace{V_{N}^{\prime}\left(x_{0}, u^{\prime}\right)-V_{N}\left(\hat{x}_{0}, u^{*}\right)}_{(*)}+\underbrace{V_{N}\left(\hat{x}_{0}, \bar{u}^{\prime}\right)-V_{N}^{\prime}\left(x_{0}, \bar{u}^{\prime}\right)}_{(* *)} .
\end{aligned}
$$

1) Bound of $(*)$ : Since $u_{0}^{\prime}=u_{0}^{*}$ we have

$$
\begin{aligned}
(*)=x_{0}^{T} Q^{\prime} x_{0}-\hat{x}_{0}^{T} Q \hat{x}_{0}+ & V_{N-1}^{\prime}\left(A^{\prime} x_{0}+B^{\prime} u_{0}^{\prime}, u^{\prime \prime}\right) \\
& -V_{N-1}\left(A \hat{x}_{0}+B u_{0}^{\prime}, u^{* *}\right),
\end{aligned}
$$

where $u^{\prime \prime}=\left(u_{1}^{\prime}, \ldots, u_{N-1}^{\prime}\right)$ and $u^{* *}=\left(u_{1}^{*}, \ldots, u_{N-1}^{*}\right)$.

Now we use the general formula

$$
V_{N}\left(x_{0}, u\right)=\left|H_{N}^{1 / 2} u+H_{N}^{-1 / 2} F_{N} x_{0}\right|^{2}+x_{0}^{T} X_{N} x_{0},
$$

where $X_{N}=\Lambda_{N}^{T} \widehat{Q}_{N} \Lambda_{N}+Q-F_{N}^{T} H_{N}^{-1} F_{N}$. We obtain that

$$
(*)=f_{A}+f_{B}+f_{C},
$$

where

$$
\begin{aligned}
f_{A}= & x_{0}^{T} Q^{\prime} x_{0}-\hat{x}_{0}^{T} Q \hat{x}_{0} \\
f_{B}= & \left|H_{N-1}^{\prime 1 / 2} u^{\prime \prime}-z\right|^{2}-\left|H_{N-1}^{1 / 2} u^{* *}-w\right|^{2} \\
f_{C}= & \left(A^{\prime} x_{0}+B^{\prime} u_{0}^{\prime}\right)^{T} X_{N-1}^{\prime}\left(A^{\prime} x_{0}+B^{\prime} u_{0}^{\prime}\right) \\
& \quad-\left(A \hat{x}_{0}+B u_{0}^{\prime}\right)^{T} X_{N-1}\left(A \hat{x}_{0}+B u_{0}^{\prime}\right) \\
& \quad-H_{N-1}^{-1 / 2} F_{N-1}\left(A \hat{x}_{0}+B u_{0}^{\prime}\right) \\
w= & -H_{N-1}^{\prime-1 / 2} F_{N-1}^{\prime}\left(A^{\prime} x_{0}+B^{\prime} u_{0}^{\prime}\right) . \\
z= &
\end{aligned}
$$

We have

$$
f_{A} \leq\left|x_{0}\right|^{2}\left\|Q-Q^{\prime}\right\|+\|Q\|\left(\left|x_{0}\right|+\left|\hat{x}_{0}\right|\right)\left|x_{0}-\hat{x}_{0}\right|,
$$

where we used the fact that for positive semidefinite $W, V$

$$
\begin{array}{r}
\left|x^{T} V x-y^{T} W y\right| \leq\left|x^{T}(V-W) x\right|+\left|x^{T} W x-y^{T} W y\right| \\
\leq\|V-W\||x|^{2}+\|W\|(|x|+|y|)|x-y| .
\end{array}
$$

If $\alpha_{\text {min }}$ is defined as in Lemma 11, then

$$
f_{A} \leq\left\|Q-Q^{\prime}\right\|\left|x_{0}\right|^{2}+\alpha_{\text {min }}\|Q\|\left(2\left|x_{0}\right|+\alpha_{\text {min }}\right) .
$$

To bound $f_{B}$, we note that $u^{\prime \prime}$ is some optimal control of $V_{N-1}^{\prime}\left(A^{\prime} x_{0}+B^{\prime} u_{0}^{\prime}, \cdot\right)$ and $u^{* *}$ is some optimal control of $V_{N-1}\left(A \hat{x}_{0}+B u_{0}^{\prime}, \cdot\right)$, that is

$$
u^{\prime \prime} \in{H_{N-1}^{\prime-1 / 2}}_{H_{N-1}^{\prime 1 / 2} \mathbb{U}^{N-1}}(z), u^{* *} \in H_{N-1}^{-1 / 2} q_{H_{N-1}^{1 / 2} \mathbb{U}^{N-1}}(w),
$$

and apply Lemma 10 to upper bound $f_{B}$ by

$$
\left(2\left\|H_{N-1}^{\prime 1 / 2}-H_{N-1}^{1 / 2}\right\| \cdot\left\|H_{N-1}^{-1 / 2}\right\| \cdot|w|+|w-z|\right)(|w|+|z|) \text {. }
$$

Furthermore, we have the following inequalities

$$
\begin{aligned}
|w-z| \leq & \left\|H_{N-1}^{\prime-1 / 2} F_{N-1}^{\prime}-H_{N-1}^{-1 / 2} F_{N-1}\right\| \cdot\left|A^{\prime} x_{0}+B^{\prime} u_{0}^{\prime}\right| \\
& +\left\|H_{N-1}^{-1 / 2} F_{N-1}\right\| \cdot\left|A^{\prime} x_{0}-A \widehat{x}_{0}+\left(B^{\prime}-B\right) u_{0}^{\prime}\right|
\end{aligned}
$$

$$
\begin{aligned}
& \left|A^{\prime} x_{0}-A \hat{x}_{0}+\left(B^{\prime}-B\right) u_{0}^{\prime}\right| \leq\left|A\left(\hat{x}_{0}-x_{0}\right)+\left(A-A^{\prime}\right) x_{0}\right| \\
& \quad+\left|B^{\prime}-B\right| \cdot\left|u_{0}^{\prime}\right| \\
& \leq \alpha_{\min }\|A\|+\left\|A-A^{\prime}\right\| \cdot\left|x_{0}\right|+\left|B^{\prime}-B\right| \cdot\left|u_{0}^{\prime}\right|=: \varepsilon_{1},
\end{aligned}
$$

and

$$
\begin{aligned}
\left|A \hat{x}_{0}+B u_{0}^{\prime}\right| & =\left|A\left(\hat{x}_{0}-x_{0}\right)+A x_{0}+B u_{0}^{\prime}\right| \\
& \leq \alpha_{\min }\|A\|+\left|A x_{0}+B u_{0}^{\prime}\right|=: d_{1},
\end{aligned}
$$

and thus $|w| \leq d_{1}\left\|H_{N-1}^{-1 / 2} F_{N-1}\right\|$.

If we define $d_{2}=\left|A^{\prime} x_{0}+B^{\prime} u_{0}^{\prime}\right|$ we see that $|z| \leq$ $d_{2}\left\|H_{N-1}^{\prime-1 / 2} F_{N-1}^{\prime}\right\|$, and if we define $T_{N}=H_{N}^{-1 / 2} F_{N}$ and $T_{N}^{\prime}=H_{N}^{\prime-1 / 2} F_{N}^{\prime}$ we have

$$
\begin{aligned}
f_{B} \leq & {\left[2 d_{1}\left\|H_{N-1}^{\prime 1 / 2}-H_{N-1}^{1 / 2}\right\| \cdot\left\|H_{N-1}^{-1 / 2}\right\| \cdot\left\|T_{N-1}\right\|\right.} \\
+ & \left.d_{2}\left\|T_{N-1}^{\prime}-T_{N-1}\right\|+\varepsilon_{1}\left\|T_{N-1}\right\|\right] \\
& \cdot\left[d_{1}\left\|T_{N-1}\right\|+d_{2}\left\|T_{N-1}^{\prime}\right\|\right] .
\end{aligned}
$$

To bound $f_{C}$, we use the fact that if $V, W, V^{\prime}, W^{\prime}$ are positive semidefinite then

$$
\begin{aligned}
& \left|y^{T}\left(V^{\prime}-W^{\prime}\right) y-x^{T}(V-W) x\right| \\
& \leq\left|x^{T} V x-y^{T} V^{\prime} y\right|+\left|x^{T} W x-y^{T} W^{\prime} y\right| \\
& \leq\left(\left\|V-V^{\prime}\right\|+\left\|W-W^{\prime}\right\|\right)|x|^{2} \\
& \quad+\left(\left\|V^{\prime}\right\|+\left\|W^{\prime}\right\|\right)(|x|+|y|)|x-y|,
\end{aligned}
$$

thus, if $L_{N}=\Lambda_{N}^{T} \widehat{Q}_{N} \Lambda_{N}$ and $W_{N}=F_{N}^{T} H_{N}^{-1} F_{N}=T_{N}^{T} T_{N}$,

$$
\begin{aligned}
f_{C} \leq & {\left[\left\|L_{N-1}-L_{N-1}^{\prime}\right\|+\left\|Q-Q^{\prime}\right\|+\left\|W_{N-1}-W_{N-1}^{\prime}\right\|\right] d_{1}^{2} } \\
& +\left[\left\|L_{N-1}^{\prime}+Q^{\prime}\right\|+\left\|W_{N-1}^{\prime}\right\|\right]\left(d_{1}+d_{2}\right) \varepsilon_{1} .
\end{aligned}
$$

2) Bound of $(* *)$ : We write $(* *)=g_{A}+g_{B}$ with

$$
\begin{aligned}
& g_{A}=V_{N}\left(\hat{x}_{0}, \bar{u}^{\prime}\right)-V_{N}^{\prime}\left(\hat{x}_{0}, \bar{u}^{\prime}\right) \\
& g_{B}=V_{N}^{\prime}\left(\hat{x}_{0}, \bar{u}^{\prime}\right)-V_{N}^{\prime}\left(x_{0}, \bar{u}^{\prime}\right)
\end{aligned}
$$

Since $\bar{u}^{\prime}$ is some optimal control of $V_{N}^{\prime}\left(x_{0}, \cdot\right)$ we have

$$
\left|H_{N}^{\prime 1 / 2} \bar{u}^{\prime}+T_{N}^{\prime} x_{0}\right| \leq\left|H_{N}^{\prime 1 / 2} v+T_{N}^{\prime} x_{0}\right|
$$

for all $v \in \mathbb{U}^{N}$, in particular $\left|H_{N}^{\prime 1 / 2} \bar{u}^{\prime}+T_{N}^{\prime} x_{0}\right| \leq\left|T_{N}^{\prime} x_{0}\right|$. Moreover

$$
\left|H_{N}^{\prime 1 / 2} \bar{u}^{\prime}+T_{N}^{\prime} \hat{x}_{0}\right| \leq\left|T_{N}^{\prime} x_{0}\right|+\left|T_{N}^{\prime}\left(x_{0}-\hat{x}_{0}\right)\right| .
$$

Thus, using (42),

$$
\begin{aligned}
& g_{B}=\left|H_{N}^{\prime 1 / 2} \bar{u}^{\prime}+T_{N}^{\prime} x_{0}\right|^{2}-\left|H_{N}^{\prime 1 / 2} \bar{u}^{\prime}+T_{N}^{\prime} \hat{x}_{0}\right|^{2} \\
& \quad+x_{0}^{T} X_{N}^{\prime} x_{0}-\hat{x}_{0}^{T} X_{N}^{\prime} \hat{x}_{0} \\
& \leq\left(2\left|T_{N}^{\prime} x_{0}\right|+\alpha_{m i n}\left\|T_{N}^{\prime}\right\|\right)\left|T_{N}^{\prime}\left(x_{0}-\hat{x}_{0}\right)\right| \\
& \quad+\left(\left\|L_{N}^{\prime}+Q^{\prime}\right\|+\left\|W_{N}^{\prime}\right\|\right)\left(\left|x_{0}\right|+\left|\hat{x}_{0}\right|\right) \alpha_{m i n} \\
& \leq \alpha_{\min }\left(2\left|x_{0}\right|+\alpha_{m i n}\right)\left(\left\|T_{N}^{\prime}\right\|^{2}+\left\|L_{N}^{\prime}+Q^{\prime}\right\|+\left\|W_{N}^{\prime}\right\|\right) .
\end{aligned}
$$

Finally,

$$
\begin{aligned}
g_{A}=\hat{x}_{0}^{T}\left(L_{N}-L_{N}^{\prime}+Q-Q^{\prime}\right) \hat{x}_{0} & +2 \bar{u}^{\prime T}\left(F_{N}-F_{N}^{\prime}\right) \hat{x}_{0} \\
& +\bar{u}^{\prime T}\left(H_{N}-H_{N}^{\prime}\right) \bar{u}^{\prime},
\end{aligned}
$$

and, since $\left|\hat{x}_{0}\right| \leq\left|x_{0}\right|+\alpha_{\min }$ and

$$
\left|\bar{u}^{\prime}\right| \leq\left\|H_{N}^{\prime-1 / 2}\right\| \cdot\left|H_{N}^{\prime 1 / 2} \bar{u}^{\prime}\right| \leq 2\left|T_{N}^{\prime} x_{0}\right| \cdot\left\|H_{N}^{\prime-1 / 2}\right\|,
$$


we see that

$$
\begin{gathered}
g_{A} \leq\left(\left|x_{0}\right|+\alpha_{m i n}\right)^{2}\left(\left\|L_{N}^{\prime}-L_{N}\right\|+\left\|Q^{\prime}-Q\right\|\right) \\
+4\left|T_{N}^{\prime} x_{0}\right| \cdot\left\|H_{N}^{\prime-1 / 2}\right\| \cdot\left\|F_{N}^{\prime}-F_{N}\right\|\left(\left|x_{0}\right|+\alpha_{m i n}\right) \\
+4\left|T_{N}^{\prime} x_{0}\right|^{2}\left\|H_{N}^{\prime-1 / 2}\right\|^{2}\left\|H_{N}^{\prime}-H_{N}\right\| .
\end{gathered}
$$

\section{REFERENCES}

[1] S. J. Qin and T. A. Badgwell, "A survey of industrial model predictive control technology," Contr. Eng. Pract., vol. 11, pp. 733-764, 2003.

[2] J. B. Rawlings and D. Q. Mayne, Model Predictive Control: Theory and Design. Madison, WI: Nob Hill Publishing, 2009.

[3] G. C. Goodwin, M. M. Serón, and J. A. De Doná, Constrained Control \& Estimation - An Optimization Perspective. London: Springer Verlag, 2005.

[4] J. M. Maciejowski, Predictive Control with Constraints. Englewood Cliffs, N. J.: Prentice-Hall, 2002.

[5] E. F. Camacho and C. Bordons, Model Predictive Control. New York, N.Y.: Springer-Verlag, 1999.

[6] P. Cortés, M. P. Kazmierowski, R. M. Kennel, D. E. Quevedo, and J. Rodríguez, "Predictive control in power electronics and drives," IEEE Trans. Ind. Electron., vol. 55, no. 12, pp. 4312-4324, Dec. 2008.

[7] P. Lezana, R. P. Aguilera, and D. E. Quevedo, "Model predictive control of an asymmetric flying capacitor converter," IEEE Trans. Ind. Electron., vol. 56, no. 6, pp. 1839-1846, June 2009.

[8] T. Geyer, G. Papafotiou, and M. Morari, "Model predictive direct torque control-part I: Concept, algorithm and analysis," IEEE Trans. Ind. Electron., vol. 56, no. 6, pp. 1894-1905, June 2009.

[9] D. E. Quevedo, H. Bölcskei, and G. C. Goodwin, "Quantization of filter bank frame expansions through moving horizon optimization," IEEE Trans. Signal Processing, vol. 57, no. 2, pp. 503-515, Feb. 2009.

[10] D. E. Quevedo, G. C. Goodwin, and J. A. De Doná, "Multistep detector for linear ISI-channels incorporating degrees of belief in past estimates," IEEE Trans. Commun., vol. 55, no. 11, pp. 2092-2103, Nov. 2007.

[11] F. Gunnarsson and F. Gustafsson, "Control theory aspects of power control in UMTS," Contr. Eng. Pract., vol. 11, pp. 1113-1125, 2003.

[12] J. A. De Doná, G. C. Goodwin, and M. M. Serón, "Anti-windup and model predictive control: Reflections and connections," European J. Contr., vol. 6, no. 5, pp. 467-477, 2000.

[13] D. E. Quevedo and G. C. Goodwin, "When is the naïve quantized control law globally optimal?" in Proc. Asian Control Conference, Melbourne, Australia, 2004, pp. 1478-1486.

[14] D. E. Quevedo, C. Müller, and G. C. Goodwin, "Conditions for optimality of naïve quantized finite horizon control," Int. J. Contr., vol. 80, no. 5, pp. 706-720, May 2007.

[15] D. E. Quevedo, G. C. Goodwin, and J. A. De Doná, "Finite constraint set receding horizon quadratic control," Int. J. Robust Nonlin. Contr., vol. 14, no. 4, pp. 355-377, Mar. 2004.

[16] G. C. Goodwin and D. E. Quevedo, "Finite alphabet control and estimation," Int. J. Contr., Automation, and Syst., vol. 1, no. 4, pp. 412 430, Dec. 2003.

[17] D. Q. Mayne, J. B. Rawlings, C. V. Rao, and P. O. M. Scokaert, "Constrained model predictive control: Optimality and stability," Automatica, vol. 36, no. 6, pp. 789-814, June 2000.

[18] A. Gersho and R. M. Gray, Vector Quantization and Signal Compression. Boston, MA: Kluwer Academic, 1992.

[19] D. E. Quevedo and G. C. Goodwin, "Multistep optimal analog-to-digital conversion," IEEE Trans. Circuits Syst. I, vol. 52, no. 4, pp. 503-515, Mar. 2005

[20] G. Ausiello, P. Crescenzi, G. Gambosi, V. Kann, A. MarchettiSpaccamela, and M. Protasi, Complexity and Approximation: Combinatorial Optimization Problems and Their Approximability Properties. Berlin: Springer-Verlag, 1998.

[21] F. Aurenhammer, "Voronoi diagrams - a survey of fundamental geometric data structure," ACM Comput. Surveys, vol. 23, no. 3, pp. 345-405, sep 1991.

[22] P. O. M. Scokaert and J. B. Rawlings, "Constrained linear quadratic regulation,” IEEE Trans. Automat. Contr., vol. 43, no. 8, pp. 1163-1169, Aug. 1998.

[23] D. Bauso, "Boolean-controlled systems via receding horizon and linear programing," Mathematics of Control, Signals, and Systems, vol. 21, no. 1 , pp. 69-91, 2009.
[24] D. Axehill, A. Hansson, and L. Vandenberghe, "Relaxations applicable to mixed integer predictive control - comparisons and efficient computations," in Proc. IEEE Conf. Decis. Contr., New Orleans, LA, 2007, pp. 4103-4109.

[25] J. P. Hespanha, D. Liberzon, and A. R. Teel, "Lyapunov conditions for input-to-state stability of impulsive systems," Automatica, vol. 44, no. 11, pp. 2735-2744, Nov. 2008.

[26] M. Nagahara and D. E. Quevedo, "Sparse representations for packetized predictive networked control," in Proc. IFAC World Congr., 2011.

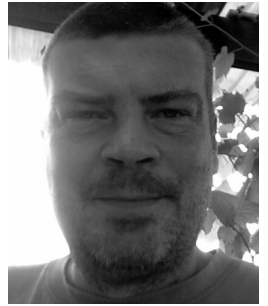

Claus Müller received a Diploma from the Technische Universität Kaiserslautern, Germany, in 1996 and the Ph.D. degree (Doktor der Naturwissenschaften) in Mathematics in 2000. After working as Assistant Professor in Kaiserslautern, he joined the School of Electrical Engineering and Computer Science at the University of Newcastle in Australia in 2003. His research topics include probability theory and functional analysis, in general, and their application to engineering problems like MPC and nonlinear filtering.

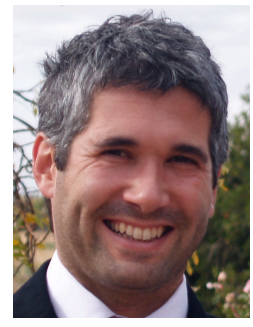

Daniel E. Quevedo (S'97-M'05) received Ingeniero Civil Electrónico and Magister en Ingeniería Electrónica degrees from the Universidad Técnica Federico Santa María, Valparaíso, Chile in 2000. In 2005, he received the Ph.D. degree from The University of Newcastle, Australia, where he is currently a research academic. He has been a visiting researcher at various Universities, including ETH Zürich, Switzerland, Uppsala University, Sweden, Aalborg University, Denmark, Kyoto University, Japan, and KTH Stockholm, Sweden.

Dr. Quevedo was supported by a full scholarship from the alumni association during his time at the Universidad Técnica Federico Santa María and received several university-wide prizes upon graduating. He received the IEEE Conference on Decision and Control Best Student Paper Award in 2003 and was also a finalist in 2002. In 2009, he was awarded an Australian Research Fellowship. His research interests cover several areas of automatic control, signal processing, communications, and power electronics.

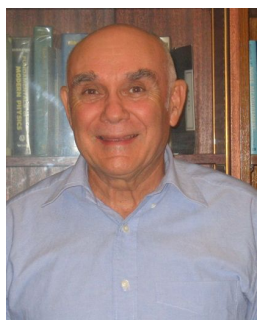

Graham C. Goodwin (M'74-SM'84-F'86) obtained a B.Sc (Physics), B.E (Electrical Engineering), and Ph.D from the University of New South Wales. He is currently Laureate Professor of Electrical Engineering at the University of Newcastle, Australia and is Director of a Priority Research Centre at the University of Newcastle. He holds Honorary Doctorates from Lund Institute of Technology, Sweden and the Technion Israel. He is the co-author of eight books, four edited books, and many technical papers.

Dr. Goodwin is the recipient of the Control Systems Society 1999 Hendrik Bode Lecture Prize, a Best Paper award by IEEE Transactions on Automatic Control, a Best Paper award by Asian Journal of Control, and 2 Best Engineering Text Book awards from the International Federation of Automatic Control in 1984 and 2005. In 2008 he received the Quazza Medal from the International Federation of Automatic Control and in 2010 he received the IEEE Control Systems Award. He is a Fellow of IEEE; an Honorary Fellow of Institute of Engineers, Australia; a Fellow of the International Federation of Automatic Control, a Fellow of the Australian Academy of Science; a Fellow of the Australian Academy of Technology, Science and Engineering; a Member of the International Statistical Institute; a Fellow of the Royal Society, London and a Foreign Member of the Royal Swedish Academy of Sciences. 